\title{
A hetero-oligomeric remorin-receptor complex regulates plant development
}

\section{Short title: A hetero-oligomeric remorin-receptor complex}

Nikolaj B. Abel ${ }^{1,2,10^{*}}$, Corinna A. Buschle ${ }^{2,11^{*}}$, Casandra Hernandez-Ryes ${ }^{1,3^{*}}$, Sandy S. Burkart $^{2,12}$, Anne-Flore Deroubaix ${ }^{4}$, Julia Mergner ${ }^{5,13}$, Julien Gronnier ${ }^{6,7}$, Iris K. Jarsch ${ }^{2}$, Jessica Folgmann², Karl Heinz Braun², Emmanuelle Bayer4, Véronique Germain ${ }^{4}$, Paul Derbyshire? Frank L.H. Menke ${ }^{7}$, Birgit Kemmerling ${ }^{8}$, Cyril Zipfel ${ }^{6,7}$, Bernhard Küster ${ }^{5,9}$, Sébastien Mongrand $^{4}$, Macarena Marín ${ }^{2}$, Thomas $\mathrm{Ott}^{1,2,3 \S}$

${ }^{1}$ Faculty of Biology, University of Freiburg, 79104 Freiburg, Germany

${ }^{2}$ Faculty of Biology, University of Munich (LMU), 82152 Planegg-Martinsried, Germany

${ }^{3}$ CIBSS - Centre for Integrative Biological Signalling Studies, University of Freiburg, 79104 Freiburg, Germany

${ }^{4}$ Laboratoire de Biogenèse Membranaire, Unité Mixte de Recherches UMR 5200, CNRS Université de Bordeaux, 33140 Villenave d'Ornon, France

${ }^{5}$ Technical University of Munich, Proteomics and Bioanalytics, 85354 Freising, Germany

${ }^{6}$ Department of Plant and Microbial Biology, Zürich-Basel Plant Science Center, University of Zürich, 8008 Zürich, Switzerland

${ }^{7}$ The Sainsbury Laboratory, University of East Anglia, Norwich Research Park, NR4 7UH, Norwich, United Kingdom

${ }^{8}$ ZMBP - Center for Plant Molecular Biology, University of Tübingen, 72076 Tübingen, Germany

9 Technical University of Munich, Bavarian Center for Biomolecular Mass Spectrometry, 85354 Freising, Germany

${ }^{10}$ present address: Aarhus University, 8000 Aarhus, Denmark

${ }^{11}$ present address: University Hospital, LMU Munich, 80337 Munich, Germany

12 present address: German Cancer Research Center (DKFZ), 69120 Heidelberg, Germany

${ }^{13}$ present address: Bavarian Center for Biomolecular Mass Spectrometry at Klinikum rechts der Isar, 81765 Munich, Germany

* these authors contributed equally to the work

$\S$ corresponding author: Thomas.Ott@biologie.uni-freiburg.de 


\section{Author contributions}

M.M., V.G., F.M., C.Z., B.K., S.M. and T.O. supervised the experiments; N.B.A., C.A.B., C.H.-R., S.N., P.D. M.M., A.-F.D., J.M., I.K.J. and J.G. performed the experiments. J.F., K.H.B. provided technical assistance to T.O.; N.B.A., C.A.B., C.H.-R., M.M., A.-F.D., J.M., J.G., V.G., S.M. and T.O. designed the experiments and analyzed the data; I.K.J., E.B. and B.K. generated and shared unpublished material. T.O. conceived the project and wrote the article with contributions of all the authors; T.O. agrees to serve as the author responsible for contact and ensures communication.

Keywords: remorin, receptor-like kinase, membrane nanodomains, plant development 


\begin{abstract}
Plant growth and development are modulated by both biotic and abiotic stress. Increasing evidence suggests that cellular integration of the corresponding signals occurs within preformed hubs at the plasma membrane called nanodomains. These membrane sub-compartments are organized by multivalent molecular scaffold proteins, such as remorins. Here, we demonstrate that Group 1 remorins form a hetero-oligomeric complex at the plasma membrane. While these remorins are functionally redundant for some pathways their multivalency also allows the recruitment of specific interaction partners. One of them, the receptor-like kinase REMORIN-INTERACTING RECEPTOR 1 (RIR1), that acts redundantly with the closely related receptor NILR2, is specifically recruited by REM1.2 in a phosphorylation-dependent manner. Overlapping developmental phenotypes suggest that the REM/RIR complex regulates key developmental pathways.
\end{abstract}




\section{Introduction}

Being exposed to a highly dynamic environment, organisms have to simultaneously integrate a plethora of extracellular stimuli. In plants, the cell wall and the plasma membrane (PM) form crucial barriers to compartmentalize, protect and modulate cytosolic processes. PM functionality depends on multiprotein entities that can form higher order assemblies in the nanometer (nanodomains) or micrometer (microdomains) range (Ott, 2017; Jaillais and Ott, 2019). Among others, membrane-resident receptors have been reported to localizes to such nanodomains ((Haney et al., 2011; Bücherl et al., 2017; Burkart and Stahl, 2017; Liang et al., 2018; Gronnier et al., 2020)). Using fluorescently-tagged fusion proteins, membrane nanodomains appear as punctate structures at the cell surface with a low degree of lateral mobility (Ott, 2017). Although precise molecular functions and mechanisms of receptor nanoclustering have only been unraveled for few cases so far (Gui et al., 2016; Liang et al., 2018) it is widely believed that these structures provide organizational units that allow protein complex formation and stabilization in complex cellular environments. Given the additional complexity of extracellular stimuli that need to be perceived and translated to generate specific cellular outputs and the comparably limited availability of multivalent proteins that could serve such functions, it can be postulated that hetero-oligomeric scaffolds such as remorin protein may act as hubs for signal integration (Jaillais and Ott, 2019).

Remorins form a multigene family with 16 members belonging to 5 different subgroups in Arabidopsis thaliana while legumes evolved an additional group (group 2) (Raffaele et al., 2007). In general, remorins are comprised of a highly diverse, N-terminal intrinsically disordered region (IDR) and a conserved coiled-coil domain-containing C-terminal segment (Raffaele et al., 2007). The latter harbors a PM binding motif (RemCA) that anchors remorins to the cytosolic leaflet of the PM, a process that is further supported by palmitoylation (Raffaele et al., 2009; Perraki et al., 2012; Konrad et al., 2014; Gronnier et al., 2017; Legrand et al., 2019). Although the molecular function remains to be elucidated for most of these proteins, recent work showed that SYMBIOTIC REMORIN 1 (SYMREM1), a group 2 remorin from Medicago truncatula, interacts with symbiotic RLKs and controls rhizobial infections by recruiting and immobilizing the LYSIN MOTIF RECEPTOR-LIKE KINASE 3 (LYK3) in specific membrane nanodomains (Lefebvre et al., 2010; Liang et al., 2018). Additional evidence for remorinmediated modulation of receptor complexes has also been presented in rice, where the group 4 remorin OsREM4.1 regulates a phytohormonal cross-talk by reversibly associating with an RLK complex in a phosphorylation-dependent manner (Gui et al., 2016). The most abundant remorins, however, belong to Group 1, with two members (REM1.2 and REM1.3) being among 
the most highly expressed proteins (Top 20\%) in Arabidopsis (Mergner et al., 2020). Even though an involvement of Group 1 remorins in phytohormone controlled processes has been suggested (Alliotte et al., 1989; Yamada et al., 1998; Demir et al., 2013; Gui et al., 2016; Huang et al., 2019), the molecular function of these proteins remains elusive. Several studies have independently demonstrated that Group 1 remorins regulate viral spreading (Raffaele et al., 2009; Son et al., 2015; Perraki et al., 2018; Cheng et al., 2020), possibly by either regulating plasmodesmatal (PD) conductance (Perraki et al., 2014; Gronnier et al., 2017; Huang et al., 2019) and/or even PD biogenesis (Wei et al., 2020). However, considering that the subcellular localization of Group 1 remorins is not limited to PDs (Raffaele et al., 2009; Jarsch et al., 2014), it appears likely that they play additional roles. Their ability to form higher order homooligomeric structures (Bariola et al., 2004; Marin et al., 2012; Legrand et al., 2019; Martinez et al., 2019) and the presence of an IDR (Marin et al., 2012) make them prominent candidates to function as multivalent scaffolds.

As such signalling hubs would be central for plant survival or at least fitness of the individuum in fluctuating environments, they can be protected by functional redundancy. To address this, we genetically analysed the hetero-oligomeric Group 1 remorin complex at the plasma membrane and demonstrate that specificity can be generated within the complex by individual members despite their overall functional redundancy. Functional specification is exemplified by the identification of the redundant receptor pair RIR1/NILR2 out of which RIR1 specifically associates with REM1.2, while NILR2 did not interact with group 1 remorins.

\section{$\underline{\text { Results }}$}

\section{Genetic redundancy within the remorin Group 1 family}

Two members of the Group 1 sub-clade, REM1.2 (At3g61260) and REM1.3 (At2g45820), belong to the top 20\% most abundant proteins in Arabidopsis (Mergner et al., 2020). While average protein levels in rosette leaves for REM1.4 (At5g23750) are almost two orders of magnitude lower compared to REM1.2 and REM1.3, the fourth Group 1 protein (REM1.1; At3g48940) was only weakly detected (in few samples and close to the detection limit) by mass spectrometry. However, low abundant transcripts of REM1.1 have been reported (Jarsch et al., 2014).

To genetically dissect functional redundancy and specificity within Group 1 remorins we used the already described rem1.2-1 and rem1.3-2 single mutant lines (Jarsch et al., 2014) and 
created a rem1.2/rem1.3 double and a rem1.2/1.3/1.4 triple mutant by introgressing the rem1.43 (N651091) allele. No knock-out allele was identified for REM1.1. None of these lines showed a significant developmental phenotype when scoring rosette diameter over a period of 28 days (Fig. 1A). As this was in contrast to previous reports (Huang et al., 2019; Wei et al., 2020), we tested the published rem 1.2/1.3c-L27 knock-out line, but did also not observe the reported growth defect under our growth conditions (Fig. S1A), while the inducible over-expressor XVE:REM1.2 was indeed growth-retarded as previously reported (Huang et al., 2019) (Fig. S1B). This indicates that the rem1.2/1.3 developmental phenotype might be conditional. Thus, we tested viral spreading as this has been reported to be altered in Group 1 remorin mutants (Raffaele et al., 2009; Perraki et al., 2018). For this, rem1.2/1.3 and rem1.2/1.3/1.4 mutants were infected with a fluorescently labelled Plantago asiatica mosaic virus (PlAMV), a Potexvirus that has previously been shown to infect $A$. thaliana (Minato et al., 2014; Hashimoto et al., 2016). Virus propagation was scored 7 dpi by determining the size of the PlAMV-GFP foci. Wild-type Col-0 plants showed average infection sites of $0.58 \mathrm{~mm}^{2}$ (Fig. 1B). While areas of PlAMV infection foci in rem 1.2 and rem 1.3 single mutants were indistinguishable from those found on WT plants, the rem1.2/1.3 double mutant was significantly more susceptible to PlAMV. This effect was further pronounced in the triple mutant (Fig. 1B). These data demonstrate that REM1.2, REM1.3 and REM1.4 act redundantly during viral spreading.

To test whether this phenomenon can also be observed for other cellular processes we made use of the fact that heterologous over-expression of the Group 1 remorin SiREM6 from foxtail millet (Setaria italica) in A. thaliana resulted in increased salt tolerance of the transgenic lines (Yue et al., 2014). Therefore, we germinated seeds on 0.5x MS plates, before we transferred seedlings onto $100 \mathrm{mM} \mathrm{NaCl}$ for seven days and scored their root lengths. While no significant differences were observed between WT and the rem1.2 and rem1.3 single mutants, the rem 1.2/1.3 double and rem 1.2/1.3/1.4 triple mutant showed a highly significant reduction in root length under salt stress (Fig. 1C). Thus, we conclude that all three REMs act redundantly in the same pathway with respect to salt tolerance.

To fully assess functional redundancy within this remorin clade, we generated quadruple rem1.1/1.2/1.3/1.4 mutant lines by CRISPR-Cas9. For this, we designed guide RNAs targeting REM1.1 with REM1.2 and REM1.4 as putative off-targets. While we were never able to generate homozygous T2 lines, we were able to rescue two lines in the T1 generation being heterozygous in the REMI.1 locus of which all individuals showed a dwarf phenotype when grown on soil (Fig. 1D). This unequivocally demonstrates that Group 1 remorins, which act at least partially redundant, are essential for plant growth but may exhibit their full functional 
potential under more natural conditions that require multi-layered integration of environmental cues.

\section{Remorins form a heteromeric and multivalent scaffolding complex at the plasma membrane}

Given their functional redundancy and the fact that remorins have already been described to form homo-oligomeric complexes (Bariola et al., 2004; Marin et al., 2012; Perraki et al., 2012; McBride et al., 2017; Martinez et al., 2019), we asked whether the highly abundant REM1.2 and REM1.3 proteins also form hetero-oligomeric complexes in A. thaliana. For this, we immunoprecipitated both fluorophore-tagged remorins expressed in their corresponding mutant backgrounds and under control of their native promoters using GFP-nanotraps and confirmed association of the endogenous REM1.2 and REM1.3 using a specific antibody against the endogenous proteins (Fig. 2A). To obtain a more global view and to dissect whether we can detect specific interaction profiles for both remorins, we immunoprecipitated REM1.2 and REM1.3 and determined associated proteins by untargeted mass-spectrometry. We obtained high confident spectra representing 2163 and 2140 unique protein identifiers found in association with REM1.2 and REM1.3, respectively with 2108 protein identifiers being identical for both remorins. However, despite this large overlap, both genotypes could clearly be separated based on their protein intensity profiles (Fig. 2B) and significant different protein interaction partners (Fig. 2C). These data support the observed genetic redundancy but also imply that specificity within the hetero-oligomeric REM complex may be generated by individual members.

\section{The membrane-resident LRR-malectin receptor RIR1 interacts with REM1.2}

Since the representation of receptors was very low in our proteome data set, aiming to identify new receptors that regulate plant growth and assuming that several of these RLKs are difficult to extract and often lowly abundant, we decided to supplement our interaction data with a targeted screen where we tested for pairwise interactions between REM1.2 and the cytoplasmic domains (CDs) of 55 different RLKs by conducting a classical yeast-2-hybrid screen (Table S1). These RLKs had originally be selected to their putative involvement in plant-microbe interactions. Using REM1.2 as bait, a single CD encoded by Atlg53440 was found to reproducibly interact with this remorin protein. Growth of yeast colonies on triple selective LWH drop out medium was sustained in all three tested dilutions and maintained in the presence of $2.5 \mathrm{mM}$ 3-Amino-1,2,4-triazole (3-AT), a competitive inhibitor of histidine biosynthesis 
(Fig. 3A). Therefore, we named the corresponding protein REMORIN-INTERACTING RECEPTOR 1 (RIR1). In this experimental system, the RIR1/REM1.2 interaction was found to be highly specific as the RIR1 CD was unable to associate with REM1.3 (Fig. 3A). Functionality and stability of both remorin clones were independently verified by testing for homo-oligomerisation (Fig. 3A) and detection of full-length proteins by Western blotting (Fig. S2).

The RIRI gene encodes a receptor-like kinase with 11 predicted leucine-rich repeats (LRRs) and a malectin-domain in its extracellular domain and a kinase domain in its C-terminal intracellular region (Fig. 3B). Previous phylogenetic analyses placed this protein into the subgroup VIII-2 of the LRR-RLK family (Shiu and Bleecker, 2001).

Since we were unable to identify RIR1 as a possible candidate from our initial MS data (Table S2) we aimed to verify this interaction in the Arabidopsis homologous system. For this, we isolated two independent SALK T-DNA insertion lines (rirl-1 (Salk_130548) and rirl-2 (Salk_057812)). Re-sequencing the insertion loci confirmed the presence of the T-DNAs at 129 base pairs (bp) upstream of the predicted translational start codon in rirl-2 and within the $21^{\text {st }}$ exon at nucleotide position 4414 in rirl-1 (Fig. 3C). Expression analysis confirmed a transcriptional knock-down for both lines (Fig. 3D). However, none of the two rirl mutant alleles showed a significant developmental phenotype (Fig. S3). Next, we introduced a RIR1GFP fusion protein driven by the native RIR 1 promoter $(1.8 \mathrm{~kb}$ upstream of the start codon; ProRIR1:RIR1-GFP) in the rir1-1 mutant background. Based on fluorescent signal intensities we selected two independent complemented lines (rir1-1/RIR1-GFP\#3 and \#4) that were used in the following analyses. Subcellular localization of RIR1-GFP in these transgenics revealed exclusive fluorescence at the plasma membrane that co-localized with the styryl dye FM4-64 (Fig. 3E). To test the RIR1-remorin interaction in planta we transformed a ProREM1.2mCherry-REM1.2 and a ProREM1.3-mCherry-REM1.3 into the rir1-1/RIR1-GFP\#3 line. Using a GFP-nanotrap, we immunoprecipitated RIR1-GFP from these lines and detected mCherry-REM1.2 and mCherry-REM1.3 co-precipitating with RIR1 (Fig. 3F). As we could not detect any interaction between REM1.3 and RIR1 in yeast, these data indicate that REM1.3 is an integral part of the RIR1-REM1.2 complex, but interacts rather with REM1.2 than with the receptor itself. To test this in planta, we used cotyledons of 5 days old homozygous seedlings to perform Fluorescence Lifetime Imaging Microscopy- Foerster Resonance Energy Transfer (FLIM-FRET). FLIM analysis on the $\operatorname{rir} 1-1 / \mathrm{RIR} 1-G F P \# 3 / \mathrm{mCherry-REM1.2-line} \mathrm{(D=}$ donor; $A=$ acceptor) indeed revealed significantly reduced lifetimes $(\tau)$ with average values of $\tau_{\mathrm{DA}} 2.21+/-0.05$ ns while the donor-only $\left(\tau_{\mathrm{D}}\right)$ lifetime of GFP in the rirl-1/RIR1-GFP\#3 line 
was $2.58+/-0.03$ nanoseconds (ns) (Fig. 4). In contrast, no decrease in fluorescence lifetime was observed in $\operatorname{rir1-1/RIR1-GFP/mCherry-REM1.3-line~with~average~} \tau_{\mathrm{DA}}$-values of 2.62 +/- 0.01 ns (Fig. 4). These data clearly support that RIR1 is recruited into the hetero-oligomeric REM1.2/REM1.3 complex where it predominantly and directly interacts with REM1.2.

\section{RIR1 localizes to membrane nanodomains independent of REM1.2/REM1.3}

Since several RLKs have been reported to localize to distinct membrane nanodomains (Haney et al., 2011; Wang et al., 2015; Bücherl et al., 2017; Hutten et al., 2017) we asked whether RIR1 also localizes to these structures at the PM. In order to minimize background fluorescence, we applied Variable Angle Total Internal Reflection Microscopy (VA-TIRFM). Analysis of the rir 1-1/RIR1-GFP\#3 line confirmed that RIR1 localizes to small nanodomains that appeared as punctate structures with high signal intensities at the cell surface (Fig. S4A). Single particle tracking (SPT) revealed an immobility of the RIR1-labelled nanodomains (Fig. S4B-D), which is a hallmark of these structures in plants. To assess whether the REM1.2/REM1.3 complex is required for the lateral immobilization of RIR1, we transformed the ProRIR1:RIR1-GFP construct into the rem1.2 single and the rem1.2/1.3 double mutant backgrounds. Analysis of homozygous lines showed neither an altered nanodomain localization pattern (Fig. S4A) nor any differences in RIR1 mobility (Fig. S4B-D) in these mutant backgrounds indicating that the REM1.2/REM1.3 scaffolding complex is not required for nanodomain recruitment of RIR1.

\section{REM1.2 and RIR1 interact in a phosphorylation-dependent manner}

Since phosphorylation of Group 1 remorins has been repeatedly reported (Benschop et al., 2007; Keinath et al., 2010; Kohorn et al., 2014; Gui et al., 2016; Menz et al., 2016; Nikonorova et al., 2018; Perraki et al., 2018), we tested whether the RIR1/REM1.2 interaction depends on this post-translational modification. For this, we created a kinase-dead variant of RIR1, where we mutated the aspartate (D) in the conserved DFG motif within the activation loop of the kinase domain into an asparagine $(\mathrm{N})\left(\mathrm{RIR} 1^{\mathrm{D} 811 \mathrm{~N}}\right)$. Mutations of the conserved aspartate are known to result in kinase-dead mutants (Yoshida and Parniske, 2005). Indeed, the D811N mutation in RIR1 entirely abolished the interaction with REM1.2 in yeast (Fig. 5A).

Since the majority of phosphorylation sites are located within the intrinsically disordered region (IDR) of Group 1 remorins (Marín and Ott, 2012), we first tested the contributions of the IDR and the structured coiled-coil C-terminal region (Cc) to RIR1/REM complex formation. Interestingly, chimeric variants of REM1.2 and REM1.3 (REM1.2 ${ }^{\text {IDR }}:$ REM1.3 ${ }^{\mathrm{Cc}}$ and REM1.3 ${ }^{\mathrm{IDR}}: \mathrm{REM} 1.2^{\mathrm{Cc}}$ ) failed to interact with RIR1 in yeast (Fig. 5B) indicating that both 
regions contribute to the interaction. Next, we created a series of phosphoablative mutations

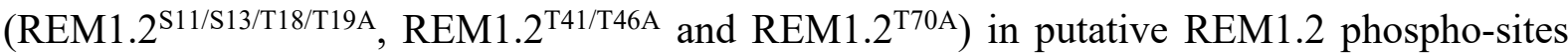
focusing on residues that are not conserved between REM1.2 and REM1.3. While the REM1.2 $2^{\mathrm{S} 11 / \mathrm{S} 13 / \mathrm{T} 18 / \mathrm{T} 19 \mathrm{~A}}$ mutant failed to interact with RIR1, the REM1.2 $2^{\mathrm{T} 41 / \mathrm{T} 46 \mathrm{~A}}$ and REM1.2 $2^{\mathrm{T} 70 \mathrm{~A}}$ mutants showed sustained yeast growth under selective conditions (Fig. 5A). Expression of all clones not yielding an interaction was confirmed by Western blot analysis (Fig. S5). These results indicate that the SSTT cluster between residues 10 and 20 within the REM1.2 N-terminal region contributes to the REM1.2/RIR1 complex formation.

\section{RIR1 is a component of a functionally redundant receptor cluster}

As mentioned above, our rirl mutant alleles did not show any significant growth defects (Fig. S3) as demonstrated for the rem1.1/1.2/1.3/1.4 quadruple mutant (Fig. 1D). To test for overlapping phenotypes between remorins and the RIR1 receptor, we inoculated rirl-1 and rir 1-2 mutants with PlAMV and scored viral infection foci after 7 days. Even though both alleles showed a slightly increased susceptibility to PlAMV, these effects were significantly smaller compared to those observed for the rem 1.2/1.3/1.4 triple mutant (Fig. 1B).

To obtain more insights into proteins interacting with RIR1, we performed immunoprecipitation followed by mass-spectrometry using the rir1-1/RIR1-GFP\#3 line and a line expressing the membrane marker LTI6b-GFP as a control. Interestingly, we identified an additional malectin-domain containing RLKs (At1g53430) directly flanking the RIR1 gene (Atlg53440), among others, as putative interactors of RIR1 (Table S3) with At1g53430 sharing about $90 \%$ sequence similarity with RIR1. However, we identified two exclusive unique peptides in two independent biological replicates and these peptides were observed with multiple spectra with good Mascot scores. In contrast, a third receptor of this gene cluster (At1G53420) does not have any exclusive unique peptides and so most spectra are likely to derive from either RIR1 or At1G53430. In addition, RIR1 and At1g53430 are expressed at similar protein levels while At1g53420 is extremely low abundant (Mergner et al., 2020); proteomicsDB: https://www.proteomicsdb.org). In analogy to RIR1, At1g53430 contains 11 LRRs followed by a malectin domain prior in the ectodomain (Fig. 7A). This RLK has been previously described to be transcriptionally induced during nematode infection and was therefore named NEMATODE-INDUCED LRR-RLK 2 (NILR2) (Mendy et al., 2017). However, a nilr2 mutant did not exhibit any susceptibility phenotype upon infection with nematodes (Mendy et al., 2017). 
To get more insight into a functional relationship between RIR1 and NILR2, we searched publicly available transcriptome datasets. Here, RIR1 and NILR2 transcripts were shown to be induced in Arabidopsis upon treatment of seedlings for 30 minutes with the bacterial elicitor flg22 (Bjornson et al., 2020), we tested transcript abundance of both receptors over a prolonged incubation period. For this, we syringe-infiltrated a $1 \mu \mathrm{M}$ flg22 solution into leaves of 5-weekold Arabidopsis plants and harvested them after 1 and 24 hours. At neither time point transcript levels of RIR1 and NILR2 were different compared to the water control (Fig. S6). However, we observed a significant reduction between 1 and 24 hours of flg22 treatment as well as in the controls. Since this effect was also found in water-infiltrated plants, we scored transcriptional changes in a detailed time course experiment. Interestingly, infiltration of water was sufficient to transiently induce $R I R 1$ and NILR2 expression, reaching a maximal expression at 1 hour post infiltration (Fig. 6A-B). As our data show that RIR1 directly interacts with the heterooligomeric REM1.2/1.3 complex, we also tested expression of these genes and found that REM1.2 and REM1.3 transcripts followed a similar pattern (Fig. 6C-D). However, expression patterns of the related malectin-domain receptor $L I K 1$, that has previously been shown to interact with the cytokinin receptor CERK1 (Le et al., 2014) and the remorin REM1.4 were distinct from RIR1, NILR2, REM1.2 and REM1.3 (Fig. 6E-F). To test whether these transcriptional inductions are an integral part of a wounding response triggered by water infiltration, we mechanically injured five weeks old rosette leaves with a needle and scored transcript abundance at one and 24 hours after wounding. No induction was found after one hour, receptor transcripts were found to be reduced (Fig. S7A-B) while REM1.2 and REM1.3 barely responded to the stimulus at all (Fig. S7C-D). In contrast, expression of the wounding marker genes JASMONATE-ZIM-DOMAIN PROTEIN 10 (JAZ10; At5g13220) and LIPOXYGENASE 3 (LOX3; At1g17420) were induced after one hour and reduced at 24 hours after wounding (Fig. S7E-F). These data indicate that the observed transcriptional inductions of RIR1, NILR2, REM1.2 and REM1.3 upon water infiltration do not represent a classical wounding response.

To test functional redundancy on a genetic level, we isolated two independent homozygous nilr2 mutant alleles (nilr2-1 (SALK_047602.23.65.n); nilr2-2 (SALK_129312C)) (Fig. 7A). Transcript analysis demonstrated only nilr2-1 to be a transcriptional null while nilr2-2 transcripts were only moderately affected (Fig. 7B). However, and in analogy to our rirl mutants we did not observe any significant developmental phenotype in any of the two alleles (Fig. 7C). 
Since chromosomal proximity of RIR1 and NILR2 did not allow the generation of double mutants by crossing, we introduced a CRISPR-CAS9 construct using four guide RNAs against RIR1 into the nilr2-1 mutant background. Here guide RNAs 87 and 14 (see Table S4) were effective and generated a 1725bp deletion between bases 1938 and 3662 of the RIR1 genomic locus. Quantitative RealTime-RT-PCR showed the absence of transcript for both genes indicating these lines to be knock-down alleles (Fig. S8). Homozygous nilr2-1/rir1 ${ }^{\text {crispr }}$ double mutants were developmentally retarded (Fig. 7C-D) and thus phenocopied the rem1.1/1.2/1.3/1.4 quadruple mutant during an early phase of development. However, this retardation was compensated over time leading to plants that were phenotypically indifferent from the WT upon flowering. These data suggest that RIR1 and NILR2 are functionally redundant. Since we were so far unable to unravel the precise molecular function of both receptors, further studies are needed to define the precise interplay and mode of action of these two receptors.

\section{$\underline{\text { Discussion }}$}

Although remorin proteins have been intensively studied in the recent past (reviewed in Gouguet et al., 2020) the precise molecular function remains elusive for most of them. However, some canonical features have been unraveled, among them their ability to from higher order oligomers (Bariola et al., 2004; Marin et al., 2012; Legrand et al., 2019; Martinez et al., 2019) and to interact with receptor-like kinases (Lefebvre et al., 2010; Gui et al., 2016; Liang et al., 2018). Among the remorin protein family, the phylogenetic Group 1 (Raffaele et al., 2007) stands out due to the high abundance of REM1.2 and REM1.3. However, it was only until recently that general growth defects have been observed for rem1.2/1.3 double mutants (Huang et al., 2019). Although we could not confirm this phenotype under our growth conditions when using the same genetic material, a higher order quadruple mutant indeed exhibited a dwarfism phenotype (Fig. 1D). Since the severity of the phenotype may depend on the growth conditions and likely to environmental stresses, it is possible that such differences explain the discrepancy between phenotypical observations of the rem1.2/1.3 double mutants. Our genetic and protein data further support the existence of an endogenous hetero-oligomeric remorin complex in A. thaliana (Fig. 2A). Both, REM1.2 and REM1.3 have a largely overlapping interactome, although they can still be clearly distinguished from each other using PCA analysis (Fig. 2B-C). Taken together these data indicate that Group 1 remorins form a 
hetero-oligomeric scaffold at the plasma membrane that serves core functions in plant growth and development and in responses to stress (e.g. salt stress and viral infections; Fig. 1).

The involvement of remorins in general plant growth by restricting plasmodesmal aperture as previously suggested (Perraki et al., 2014; Gronnier et al., 2017; Huang et al., 2019) was confirmed using Arabidopsis double mutants (Fig. 1B). The growth retarded phenotype of the rir1/nilr2 double mutant during early seedling development (Fig. 7) places both receptors into this genetic pathway, although a precise role remains to be unraveled. The genetic interaction between RIR1 and NILR2 is also supported by a recent in silico receptor interaction network analysis that indicated an association of RIR1, NILR2 and a so far uncharacterized LRR-RLK (At5g01950) (Xi et al., 2019). Interestingly, the phylogentically related MD-receptor-like kinase LMK1 (At1g07650) was reported to function as a cell death-related receptor as it induced cell death when ectopically expressed in N. benthamiana leaf epidermal cells (Li et al., 2020). While we did not observe such phenomenon for RIR1, we were indeed also unable to over-express NILR2 in these cell types (data not shown). Possible detrimental effects due to ectopic expression in their homologous plant backgrounds have also been reported for Arabidopsis (Huang et al., 2019); Fig. S1B) and Solanum lycopersicum (Cai et al., 2020) remorin proteins. In addition, we have not succeeded generating stable transgenic Arabidopsis lines constitutively over-expressing group 1 remorins at high level.

The presence of an MD in both receptors indicates possible functions in sensing cell wall integrity as an animal malectin protein has been shown to bind Glc2-N-glycans (Schallus et al., 2008; Schallus et al., 2010). Besides MDs, some plant RLKs like the CrRLK1Ls contain two malectin-like domains (MLD) in a tandem organization in their extracellular region (BoissonDernier et al., 2011). Members of this family are thought to bind to the cell wall and act as sensors of cell wall integrity (Cheung and Wu, 2011). In line with this, several CrRLK1Ls have been shown to be involved in various growth- and stress-related pathways (Galindo-Trigo et al., 2016). Mutations in the RLK THESEUS1 (THE1), for example, were demonstrated to partially restore growth defects of the cellulose-deficient mutant prcl-1, without altering the cellulose content (Hématy et al., 2007). In addition, a functional THE1 is needed for the oxidative burst triggered by the cellulose synthesis inhibitor isoxaben (Denness et al., 2011). However, it was recently demonstrated that MLDs can bind RALF peptides and that they lack key residues required for direct carbohydrate binding (Xiao et al., 2019).

Since a huge variety of environmental stimuli needs to be integrated simultaneously and given the limited capability of lateral diffusion of membrane proteins in plant cells (Martinière et al., 2019), scaffolding proteins could dynamically facilitate and compartmentalize these processes. 
However, RIR1 mobility and its recruitment to membrane nanodomains is not altered in remorin mutants (Fig. S4), indicating REM1.2 and REM1.3 do not regulate lateral diffusion of RIR1 at the PM itself. This is in contrast to the group 2 remorin SYMREM1 from Medicago truncatula that immobilizes the entry receptor LYK3 in membrane nanodomains during symbiotic interaction with rhizobia (Liang et al., 2018). However, it was recently shown that the presence of Group 1 remorins can alter membrane fluidity (Huang et al., 2019) and as a consequence the dynamic behavior of adjacent membrane proteins. Whether this is also the case for FLS2 that has been shown to partially co-localize in REM1.2/REM1.3 positive membrane nanodomains (Bücherl et al., 2017) remains to be demonstrated. However, we hypothesize at this stage that the transient transcriptional induction of Group 1 remorins upon infiltration of water into leaves (Fig. 7) may be part of a cellular response to minimize membrane damage under these conditions. It would indeed be interesting to test, whether the ability of remorins to form highly oligomeric proteinaceous leaflets (Martinez et al., 2018) or potentially liquid-liquid-phase separates (LLPS) (Jaillais and Ott, 2019) may assist membrane stabilization under conditions of negative curvature and membrane tension that cannot be compensated by the rigid cell wall. Given the presence of the malectin domain in RIR1 and NILR2 (Fig. 3B, 7A), these receptors might act as sensors of PM-cell wall proximity and get activated upon retraction of the PM from the cell wall.

\section{Material and Methods}

\section{Plant materials and growth conditions}

T-DNA insertion mutants of REM1.2 (At3g61260) rem1.2-2 (SALK_117637.50.50.x), REM1.3 (At2g45820) rem1.3-2 (SALK_117448.53.95.x) and REM1.4 (At5g23750.x) rem 1.43 (SALK_073841.47.35), rir1-1 (Salk_130548.42.45.x), rirl-2 (Salk_057812.14.90.x) were provided by the ABRC. rem1.2-2/rem1.3-2 (rem1.2/rem1.3) double mutant was generated by crossing the respective T-DNA inserted parental plants, rem1.2-2/rem1.3-2/rem 1.4-3 (rem1.2/rem1.3/rem1.4) was created by crossing rem1.2/rem1.3 with rem1.4-3. All plants were genotyped using primers indicated in Table S4.

ProREM1.2:YFP-REM1.2 and ProREM1.3:YFP-REM1.3 lines were described previously (Jarsch et al., 2014).

Surface-sterilized $A$. thaliana seeds were germinated on $1 / 2$ MS plates supplemented with $1 \%$ sucrose. If not indicated differently seedlings were transferred to soil and grown under short 
day conditions ( $8 \mathrm{~h}$ light/ $16 \mathrm{~h}$ dark). For immunoprecipitation followed by mass spectrometry, seeds of indicated lines were sterilized and germinated on metal grids placed on solid $1 / 2 \mathrm{MS}$ media and grown for 1 week. Metal grid including germinated seedlings were moved to liquid $1 / 2$ MS media and grown for 3 weeks under long day conditions, liquid media was replaced with fresh media every week.

The ProRIR1:RIR1-GFP construct (in pGWB604) was transformed into rir1-1, rem 1.2-1 and rem1.2-2/rem1.3-2 mutant plants by the floral dipping method ((Clough and Bent 1998)). The ProREM1.2-mCherry-gREM1.2 and ProREM1.2-mCherry-gREM1.3 constructs (in pGWB1) were additionally dipped into homozygous ProRIR1:RIR1-GFP/rir1-1 plants.

Nicotiana benthamiana plants were grown for 4 weeks under long day conditions (16 h light/ $8 \mathrm{~h}$ dark) prior to Agrobacterium-mediated transformation.

\section{Bacteria strains}

All cloning was performed using E. coli Top10 strains employing standard protocols for transformation, cultivation and lysis. Electro competent Agrobacterium tumefaciens AGL1 was used for Arabidopsis transformation.

\section{Developmental phenotyping}

Seedlings designated for examination of germinations rates and rosette diameter at 14 days were grown on vertical plates under long day conditions. The phenotyping of later development stages was performed on adult plant grown under long day conditions in the greenhouse.

\section{Salt stress phenotyping}

Seeds of indicated genotypes were sterilized then germinated on $1 / 2$ MS plates and grown for 1 week before transfer to new plates including $100 \mathrm{mM} \mathrm{NaCl}$ or mock, seedlings were grown for 1 week on $\mathrm{NaCl}$ plates before root elongation was measured.

\section{Viral spreading experiments}

PlAMV propagation experiments were performed as previously described (Gronnier et al., 2017) with minor modifications. Agrobacterium tumefaciens strain GV3101 carrying pLI1 evec-nGFP, which expresses PlAMV-GFP (Minato et al., 2014) was infiltrated on 4-weekold Arabidopisis thaliana plants grown in a greenhouse, with a final OD600 $\mathrm{nm}=0.2$. Viral spreading was tracked on inoculated leaves using Axiozoom macroscope system (Zeiss, 
Germany) at 7 DPI. The area of PlAMV-GFP infection foci was measured using Fiji software (http://www.fiji.sc/).

\section{Quantitative real-time PCR}

RNA from 10 days old seedlings were extracted using a Spectrum Plant Total RNA Kit (Sigma). DNA was removed by DNase I Amplification Grade (Invitrogen). cDNA was generated using a qScript cDNA SuperMix (Quanta bio). qPCR was performed with a Fast SYBR kit (Applied Biosystems) on a StepOnePlus Real-Time PCR system machine (Applied Biosystems) or a 96-well Real-Time PCR machine (Bio-Rad, CFX96).

Two technical and at least 3 biological replicates were used. All transcripts were normalized to the housekeeping gene AtUbiquitin $C(U B C)$. All primers used can be found in Supplemental Table S4.

For assessing gene expression under different conditions, five-week-old Arabidopsis leaves were used. Leaves were infiltrated with water or $1 \mu \mathrm{M}$ flg22 and water (as control) for the examination of the defense marker genes or wounded with a needle to analyze the stress induction. Here, non-infiltrated and non-wounded leaves were used as controls.

\section{Yeast two-hybrid assay}

Yeast two-hybrid interaction assays and expression analyses were performed as previously described (Marin et al., 2012). In brief, using the vectors pGADT7:GW and pGBKT7:GW, activation domain (AD)- and binding domain (BD)-fused constructs were co-transformed into the yeast strain PJ69-4a, respectively. Transformants were plated onto different SD plates lacking tryptophan, leucine and histidine (+/- 3-amino-1,2,4-triazole) to examine the interaction. The expression of all proteins was verified by Western Blot analysis using tagspecific antibodies.

\section{Confocal laser-scanning microscopy}

For microscopic analyses, $A$. thaliana leaves were infiltrated with $\mathrm{H}_{2} \mathrm{O}$ prior to harvest (puncher $4 \mathrm{~mm}$ diameter). Leaf discs were mounted on glass slides and immediately imaged using Leica TCS SP5 and SP8 confocal microscopes. GFP was excited with the Argon laser (AR) at 488 $\mathrm{nm}$ and the emission was detected between 500 and $550 \mathrm{~nm}$. YFP RICKY1 was excited with a wavelength of $514 \mathrm{~nm}(\mathrm{AR})$ and the emission was detected at $525-599 \mathrm{~nm}$. mCherry was excited at $561 \mathrm{~nm}$ using the Diode Pumped Solid State (DPSS) laser and the emission was 
detected at $570-640 \mathrm{~nm}$. Samples, co-expressing two fluorophores, were imaged using the sequential mode between frames.

\section{Fluorescence lifetime imaging (FLIM)}

Cotyledons of 5 days old Arabidopsis seedlings (rir1-1/ProRIR1:RIR1-GFP, rirl1/ProRIR1:RIR1-GFP/ProREM1.2:mCherry-gREM1.2 and rir1-1/ProRIR1: RIR1GFP/ProREM1.3:mCherry-gREM1.3) were used for FLIM experiments. Experiments were performed using a Leica TCS SP8X confocal microscope equipped with TCSPC (time correlated single photon counting) electronics (PicoHarp 300), photon sensitive detectors (HyD SMD detector) and a pulsed white light laser. The abaxial side of cotyledons were imaged using a 63x/1.2 water immersion objective lens (Leica C-APOCHROMAT 63×/1.2 water). GFP was excited at $488 \mathrm{~nm}$ and fluorescence emission collected between 500-540nm. The FLIM data sets were recorded using the Leica LASX FLIM wizard linked to the PicoQuant SymPhoTime 64 software and acquired by repetitively scanning (40 times) region of $70.29 \mu \mathrm{m} 2$ at $50 \mathrm{Mhz}$ with a pixel dwell time of $9.75 \mu \mathrm{s}$. After each FLIM acquisition effective expression of REM1.2 and REM1.3 was recorded by exciting mCherry at $580 \mathrm{~nm}$ and collection of corresponding fluorescence emission between 590 and $640 \mathrm{~nm}$. To visualize potential FRET, samples were excited at $488 \mathrm{~nm}$ and fluorescence emission was collected between 590 and $640 \mathrm{~nm}$. The instrument response function (IRF) was measured using erythrosine B as described (Stahl et al., 2013). Calculations of fluorescence lifetime were performed using the PicoQuant SymPhoTime 64 software following instructions for FLIM-FRET-Calculation for MultiExponential Donors. A two-exponential decay fit was used for GFP. The lifetimes were initially estimated by fitting the data using the Monte Carlo method and then by fitting the data using the Maximum Likely Hood Estimation.

\section{Total Internal Reflection Fluorescence (TIRF) microscopy}

TIRF microscopy was performed using a Zeiss Elyra PS1 (Zeiss, Germany) microscope using a $100 \times 1.4$ NA oil immersion objective. GFP was excited using a $488 \mathrm{~nm}$ solid-state laser diode and corresponding fluorescence emission was collected with an EM-CCD camera with bandwidth filters ranging from $495-550 \mathrm{~nm}$. The optimum critical angle was determined as giving the best signal-to-noise. Images time series were recorded at 10 frames per second (100 ms exposure time). To analyze single particle tracking experiments, we used the plugin TrackMate 2.7.4 (Tinevez et al., 2016) in Fiji (Schindelin et al., 2012) as previously described (Gronnier et al., 2020). 


\section{Total protein extraction:}

Tissue from Arabidopsis were harvested in equal amount and frozen in liquid nitrogen before grounded to power by a tissue lyser (for small amount) or mortar and pestle (for larger amounts). Proteins were extracted in a buffer containing: $50 \mathrm{mM}$ Tris- $\mathrm{HCl}(\mathrm{pH} 7.5), 150 \mathrm{mM}$ $\mathrm{NaCl}, 10$ \% glycerol, 2 mM EDTA 5 mM DTT, 1 mM PMSF in 2-propanol, 1.5 mM Na3VO4, $1 \mathrm{x}$ Protease Inhibitor Cocktail (Roche) and $1 \%$ (v/v) IGEPAL CA-630 (Sigma-Aldrich). Extraction was incubated for 1 hour at $4^{\circ} \mathrm{C}$ on a rotor. After incubation extracts were centrifuged at 13,000 rpm for $30 \mathrm{~min}$. Supernatants were mixed with SDS loading buffer and boiled for 5 $\min$.

\section{Immuno-precipitation mass spectrometry}

For determining remorin interactions, tissue from Arabidopsis were harvested in equal amount and frozen in liquid nitrogen before grounded to power by a tissue lyser. Proteins were extracted in a buffer containing: $50 \mathrm{mM}$ Tris- $\mathrm{HCl}(\mathrm{pH} 7.5), 150 \mathrm{mM} \mathrm{NaCl}, 10 \%$ glycerol, 2 mM EDTA $5 \mathrm{mM}$ DTT, $1 \mathrm{mM}$ PMSF in 2-propanol, $1.5 \mathrm{mM} \mathrm{Na}_{3} \mathrm{VO}_{4}, 1 \times$ Protease Inhibitor Cocktail (Roche) and $1 \%$ (v/v) IGEPAL CA-630 (Sigma-Aldrich). Extraction was performed for 1 hour at $4^{\circ} \mathrm{C}$ on a rotor. After incubation extracts were centrifuged at $13,000 \mathrm{rpm}$ for $30 \mathrm{~min}$. Supernatants were incubated with GFP-Trap (Chromotek) for 2 hours at $4^{\circ} \mathrm{C}$ on a rotor before magnetic separation and washing 5 times with washing buffer $(50 \mathrm{mM}$ Tris- $\mathrm{HCl}(\mathrm{pH} 7.5), 150$ $\mathrm{mM} \mathrm{NaCl}, 10 \%$ glycerol, $2 \mathrm{mM}$ EDTA 5 mM, 1 x Protease Inhibitor Cocktail (Roche) and 0.5 $\%$ (v/v) IGEPAL CA-630 (Sigma-Aldrich)). Extracted proteins were released with 2xNuPage LDS sample buffer and boiled for $5 \mathrm{~min}$.

Samples were reduced with $10 \mathrm{mM}$ ditiothreitol (DTT) for one hour followed by alkylation with $55 \mathrm{mM}$ chloroacetamide (CAA) for 30min at room temperature and run into a $4-12 \%$ NuPAGE gel (Invitrogen) for approximately $1 \mathrm{~cm}$. In-gel digestion was performed according to standard procedures with trypsin (Roche) (Shevchenko et al., 2006). MS measurements were performed on a Q Exactive HF-X (Thermo Fisher Scientific) using a 60 min linear gradient. The instrument was operated in data-dependent mode, automatically switching between MS and MS2 scans. Full-scan mass spectra (m/z 360-1300) were acquired in profile mode with 60,000 resolution, an automatic gain control (AGC) target value of $3 \mathrm{e} 6$ and $45 \mathrm{~ms}$ maximum injection time. For the top 20 precursor ions, high resolution MS2 scans were performed using HCD fragmentation with $28 \%$ normalized collision energy, 30,000 resolution, an AGC target 
value of $2 \mathrm{e} 5,50 \mathrm{~ms}$ maximum injection time and $1.3 \mathrm{~m} / \mathrm{z}$ isolation width in centroid mode. The minimum AGC target value was set to $2.2 \mathrm{e} 3$ with a dynamic exclusion of $25 \mathrm{~s}$.

Peptide and protein identification and quantification was performed with MaxQuant (Cox and Mann, 2008) using standard settings (version 1.6.3.3). Raw files were searched against an Arabidopsis database (Araport11_genes.201606.pep.fasta) and common contaminants. Carbamidomethylated cysteine was set as fixed modification and oxidation of methionine, and $\mathrm{N}$-terminal protein acetylation as variable modifications. Trypsin/P was specified as the proteolytic enzyme, with up to two missed cleavage sites allowed. The match between run and LFQ functions were enabled and results filtered to 1\% PSM and protein FDR. LFQ proteinGroup intensities were filtered for identifications in all three replicates of at least one group $(\mathrm{n}=2,195)$, missing values were imputed from a normal distribution and statistical analyses were performed using Perseus (v. 1.6.0.2) (Tyanova et al., 2016).

To assess RIR1 interactions, extracts of 5- to 6-week-old $A$. thaliana plants were incubated with 50-100 $\mu$ previously washed 50\% GFP-binding protein beads slurry (Chromotek, GFP-Trap ${ }^{\circledR}$ ) at $4{ }^{\circ} \mathrm{C}$ for 3 hours on a roller mixer. Samples were centrifuged to collect beads; supernatants were discarded and the beads were washed 5 times using extraction buffer containing $0.5 \%$ (v/v) IGEPAL CA-630 (Sigma-Aldrich). To release the proteins, 50-100 $\mu 1$ 2x NuPAGE LDS sample buffer (Invitrogen) was added and samples were boiled for 5 min at $95{ }^{\circ} \mathrm{C}$.

Obtained proteins were separated by SDS-PAGE (NuPAGE, Invitrogen) and after staining with Coomassie brilliant Blue G- ds were excised from the gel and transferred to LoBind tubes (Eppendorf). For in-gel digestion, gel pieces were washed twice for $30 \mathrm{~min}$ with Acetonitrile/Ammonium Bicarbonate $(\mathrm{ACN} / \mathrm{ABC})$ at $56{ }^{\circ} \mathrm{C}$ and dehydrated for $5 \mathrm{~min}$ using $100 \%$ Acetonitrile at room temperature. Supernatants were discarded and di-sulphide bonds were reduced using $10 \mathrm{mM}$ DTT in $\mathrm{ABC}$ for $45 \mathrm{~min}$ at $56^{\circ} \mathrm{C}$. For alkylation, samples were incubated for 30 min with $55 \mathrm{mM}$ Chloroacetamide in ABC in the dark. Samples were again washed and dehydrated as described before. In-gel digest was performed with trypsin (Promega) in $50 \mathrm{mM} \mathrm{ABC}$ and $5 \% \mathrm{ACN}$ at $37^{\circ} \mathrm{C}$ overnight. Peptides were recovered with 5 $\%$ Formic acid in $50 \%$ ACN by repeated sonication. Supernatants were collected in new LoBind tubes and dried completely in speed vacuum for 3 hours at room temperature. LCMS/MS analysis was performed using an Orbitrap Fusion mass-spectrometer (Thermo Scientific). The entire TAIR10 database was searched (www.Arabidopsis.org) using Mascot (v 2.3.02, Matrix Science) with the inclusion of sequences of common contaminants, such as keratins and trypsin. Parameters were set for $5 \mathrm{ppm}$ peptide mass tolerance and allowing for 
Met oxidation and two missed tryptic cleavages. Carbamidomethylation of Cys residues was specified as a fixed modification, and oxidized Met and phosphorylation of Ser, Tyr or Thr residues were allowed as variable modifications. Scaffold (v3; Proteome Software) was used to validate MS/MS-based peptide and protein identifications and annotate spectra.

\section{SDS-PAGE and Western blots}

Extracted proteins were separated on a $12 \%$ SDS-PAGE gel for $15 \mathrm{~min}$ at $80 \mathrm{~V}$ followed by 2$3 \mathrm{H}$ at $120 \mathrm{~V}$. Separated proteins were transferred to PVDF membranes at $30 \mathrm{~V}$ for $12-16 \mathrm{H}$ at $4^{\circ} \mathrm{C}$. PVDF membranes were blocked with $5 \%$ milk followed by an incubation with first antibodies for REM1.2/1.3 followed by 3 washes and an incubation with second $\alpha$-rabbit antibody fused to HRP against rabbit (Sigma). Clarity Western 701 ECL (Bio-Rad) was employed for Chemiluminescence detection. 


\section{Acknowledgments}

This project was supported by the German Research Foundation (Deutsche Forschungsgemeinschaft; DFG) in frame of the Collaborative Research Center 924 (SFB 924; T.O.), under Germany's Excellence Strategy/Initiative (CIBSS - EXC-2189 - Project ID 390939984; T.O.), supplemented by an Exploration Grant of the Boehringer Ingelheim Foundation (to T.O.), an individual DFG grant KE1485 (to B.K.), the Gatsby Charitable Foundation (C.Z.), the University of Zürich (C.Z.), the European Research Council under the Grant Agreements 309858 and 773153 (grants PHOSPHinnATE and IMMUNO-PEPTALK to C.Z.) and the Swiss National Science Foundation (grant no. 31003A_182625 to C.Z.). J.G. was supported by the European Molecular Biology Organization (EMBO Long-Term Fellowship 438-2018). We thank the staff of the Life Imaging Center (LIC) in the Center for Biological Systems Analysis (ZBSA) of the Albert-Ludwigs-University Freiburg for help with their confocal microscopy resources, and the excellent support in image recording. The CLSM Leica SP8 was funded by the DFG grant INST 39/1104-1 FUGG.

Furthermore, we would like to thank Johannes Stuttmann for discussions and for providing the CRISPR-Cas9 vectors, Eija Schulze for the great technical assistance, Elisabeth Lichtenberg and Jacqueline Cornean for their help and all members of our team for fruitful discussions and providing their individual expertise throughout the course of the project. J.G. is also thankful to Grant Calder (JIC Bioimaging facility) for assistance in setting up FLIM-FRET experiments. GFP-PIAMV viral spreading was performed at the Bordeaux Imaging Center, member of infrastructure France Bioimaging. 


\section{$\underline{\text { References }}$}

Alliotte T, Tire C, Engler G, Peleman J, Caplan A, Montagu MV, Inze D (1989) An AuxinRegulated Gene of Arabidopsis thaliana Encodes a DNA-Binding Protein. Plant Physiol 89: $743-52$

Bariola PA, Retelska D, Stasiak A, Kammerer RA, Fleming A, Hijri M, Frank S, Farmer EE (2004) Remorins form a novel family of coiled coil-forming oligomeric and filamentous proteins associated with apical, vascular and embryonic tissues in plants. Plant Mol Biol 55: 579-94

Benschop JJ, Mohammed S, O’Flaherty M, Heck AJ, Slijper M, Menke FL (2007) Quantitative phosphoproteomics of early elicitor signaling in Arabidopsis. Mol Cell Proteomics 6: 1198-214

Bjornson M, Pimprikar P, Nürnberger T, Zipfel C (2020) The transcriptional landscape of Arabidopsis thaliana pattern-triggered immunity. Biorxiv 2020.11.30.404566

Boisson-Dernier A, Kessler SA, Grossniklaus U (2011) The walls have ears: the role of plant CrRLK1Ls in sensing and transducing extracellular signals. J Exp Bot 62: 1581-1591

\section{Bücherl CA, Jarsch IK, Schudoma C, Segonzac C, Mbengue M, Robatzek S, MacLean D,} Ott T, Zipfel C (2017) Plant immune and growth receptors share common signalling components but localise to distinct plasma membrane nanodomains. eLife 6: e25114

Burkart RC, Stahl Y (2017) Dynamic complexity: plant receptor complexes at the plasma membrane. Curr Opin Plant Biol 40: 15-21

Cai J, Chen T, Wang Y, Qin G, Tian S (2020) SlREM1 Triggers Cell Death by Activating an Oxidative Burst and Other Regulators. Plant Physiol 183: 717-732

Cheng G, Yang Z, Zhang H, Zhang J, Xu J (2020) Remorin interacting with PCaP1 impairs Turnip mosaic virus intercellular movement but is antagonised by VPg. New Phytol 225: $2122-2139$

Cheung AY, Wu H-M (2011) THESEUS 1, FERONIA and relatives: a family of cell wallsensing receptor kinases? Curr Opin Plant Biol 14: 632-641 
Cox J, Mann M (2008) MaxQuant enables high peptide identification rates, individualized p.p.b.-range mass accuracies and proteome-wide protein quantification. Nat Biotechnol 26: $1367-1372$

Demir F, Horntrich C, Blachutzik JO, Scherzer S, Reinders Y, Kierszniowska S, Schulze WX, Harms GS, Hedrich R, Geiger D, et al (2013) Arabidopsis nanodomain-delimited ABA signaling pathway regulates the anion channel SLAH3. Proc National Acad Sci 110: $8296-301$

Denness L, McKenna JF, Segonzac C, Wormit A, Madhou P, Bennett M, Mansfield J, Zipfel C, Hamann T (2011) Cell Wall Damage-Induced Lignin Biosynthesis Is Regulated by a Reactive Oxygen Species- and Jasmonic Acid-Dependent Process in Arabidopsis. Plant Physiol 156: 1364-1374

Galindo-Trigo S, Gray JE, Smith LM (2016) Conserved Roles of CrRLK1L Receptor-Like Kinases in Cell Expansion and Reproduction from Algae to Angiosperms. Front Plant Sci 07: 1269

Gronnier J, Crowet JM, Habenstein B, Nasir MN, Bayle V, Hosy E, Platre MP, Gouguet P, Raffaele S, Martinez D, et al (2017) Structural basis for plant plasma membrane protein dynamics and organization into functional nanodomains. eLife 6: e26404

Gronnier J, Franck CM, Stegmann M, DeFalco TA, Cifuentes AA, Dünser K, Lin W, Yang Z, Kleine-Vehn J, Ringli C, et al (2020) FERONIA regulates FLS2 plasma membrane nanoscale dynamics to modulate plant immune signaling. Biorxiv 2020.07.20.212233

Gui J, Zheng S, Liu C, Shen J, Li J, Li L (2016) OsREM4.1 Interacts with OsSERK1 to Coordinate the Interlinking between Abscisic Acid and Brassinosteroid Signaling in Rice. Developmental cell 38: 201-13

Haney CH, Riely BK, Tricoli DM, Cook DR, Ehrhardt DW, Long SR (2011) Symbiotic Rhizobia Bacteria Trigger a Change in Localization and Dynamics of the Medicago truncatula Receptor Kinase LYK3. Plant Cell 23: 2774-2787

Hashimoto M, Neriya Y, Keima T, Iwabuchi N, Koinuma H, Hagiwara-Komoda Y, Ishikawa K, Himeno M, Maejima K, Yamaji Y, et al (2016) EXA1, a GYF domain 
protein, is responsible for loss-of-susceptibility to plantago asiatica mosaic virus in Arabidopsis thaliana. Plant J 88: 120-131

Hématy K, Sado P-E, Tuinen AV, Rochange S, Desnos T, Balzergue S, Pelletier S, Renou J-P, Höfte H (2007) A Receptor-like Kinase Mediates the Response of Arabidopsis Cells to the Inhibition of Cellulose Synthesis. Curr Biol 17: 922-931

Huang D, Sun Y, Ma Z, Ke M, Cui Y, Chen Z, Chen C, Ji C, Tran TM, Yang L, et al (2019) Salicylic acid-mediated plasmodesmal closure via Remorin-dependent lipid organization. Proc National Acad Sci 116: 21274-21284

Hutten SJ, Hamers DS, Toorn MA den, Esse W van, Nolles A, Bucherl CA, Vries SC de, Hohlbein J, Borst JW (2017) Visualization of BRI1 and SERK3/BAK1 Nanoclusters in Arabidopsis Roots. PLOS ONE 12: e0169905

Jaillais Y, Ott T (2019) The nanoscale organization of the plasma membrane and its importance in signaling - a proteolipid perspective. Plant Physiol 182: pp.01349.2019

Jarsch IK, Konrad SS, Stratil TF, Urbanus SL, Szymanski W, Braun P, Braun KH, Ott T (2014) Plasma Membranes Are Subcompartmentalized into a Plethora of Coexisting and Diverse Microdomains in Arabidopsis and Nicotiana benthamiana. Plant Cell 26: 16981711

Keinath NF, Kierszniowska S, Lorek J, Bourdais G, Kessler SA, Shimosato-Asano H, Grossniklaus U, Schulze WX, Robatzek S, Panstruga R (2010) PAMP (pathogenassociated molecular pattern)-induced changes in plasma membrane compartmentalization reveal novel components of plant immunity. J Biol Chem 285: 39140-9

Kohorn BD, Kohorn SL, Saba NJ, Martinez VM (2014) Requirement for pectin methyl esterase and preference for fragmented over native pectins for wall-associated kinaseactivated, EDS1/PAD4-dependent stress response in Arabidopsis. J Biol Chem 289: 1897886

Konrad SS, Popp C, Stratil TF, Jarsch IK, Thallmair V, Folgmann J, Marin M, Ott T (2014) S-acylation anchors remorin proteins to the plasma membrane but does not primarily determine their localization in membrane microdomains. New Phytol 203: 758-69 
Le MH, Cao Y, Zhang XC, Stacey G (2014) LIK1, a CERK1-interacting kinase, regulates plant immune responses in Arabidopsis. PLOS ONE 9: e102245

\section{Lefebvre B, Timmers T, Mbengue M, Moreau S, Herve C, Toth K, Bittencourt-Silvestre} J, Klaus D, Deslandes L, Godiard L, et al (2010) A remorin protein interacts with symbiotic receptors and regulates bacterial infection. Proc Natl Acad Sci U S A 107: 23438

Legrand A, Martinez D, Grélard A, Berbon M, Morvan E, Tawani A, Loquet A, Mongrand S, Habenstein B (2019) Nanodomain Clustering of the Plant Protein Remorin by Solid-State NMR. Frontiers Mol Biosci 6: 107

\section{Li X, Sanagi M, Lu Y, Nomura Y, Stolze SC, Yasuda S, Saijo Y, Schulze WX, Feil R, Stitt} M, et al (2020) Protein Phosphorylation Dynamics Under Carbon/Nitrogen-Nutrient Stress and Identification of a Cell Death-Related Receptor-Like Kinase in Arabidopsis. Front Plant Sci 11: 377

Liang P, Stratil TF, Popp C, Marin M, Folgmann J, Mysore KS, Wen J, Ott T (2018) Symbiotic root infections in Medicago truncatula require remorin-mediated receptor stabilization in membrane nanodomains. Proc National Acad Sci 115: 5289-5294

Marín M, Ott T (2012) Phosphorylation of Intrinsically Disordered Regions in Remorin Proteins. Frontiers in Plant Sciences 3: 86

Marin M, Thallmair V, Ott T (2012) The intrinsically disordered N-terminal region of AtREM1.3 remorin protein mediates protein-protein interactions. J Biol Chem 287: 3998291

Martinez D, Legrand A, Gronnier J, Decossas M, Gouguet P, Lambert O, Berbon M, Verron L, Grélard A, Germain V, et al (2019) Coiled-coil oligomerization controls localization of the plasma membrane REMORINs. J Struct Biol 206: 12-19

Martinière A, Fiche JB, Smokvarska M, Mari S, Alcon C, Dumont X, Hematy K, Jaillais Y, Nollmann M, Maurel C (2019) Osmotic Stress Activates Two Reactive Oxygen Species Pathways with Distinct Effects on Protein Nanodomains and Diffusion. Plant Physiol 179: 1581-1593 
McBride Z, Chen D, Reick C, Xie J, Szymanski DB (2017) Global Analysis of Membraneassociated Protein Oligomerization Using Protein Correlation Profiling*. Mol Cell Proteomics 16: 1972-1989

Mendy B, Wang'ombe MW, Radakovic ZS, Holbein J, Ilyas M, Chopra D, Holton N, Zipfel C, Grundler FMW, Siddique S (2017) Arabidopsis leucine-rich repeat receptorlike kinase NILR1 is required for induction of innate immunity to parasitic nematodes. Plos Pathog 13: e1006284

Menz J, Li Z, Schulze WX, Ludewig U (2016) Early nitrogen-deprivation responses in Arabidopsis roots reveal distinct differences on transcriptome and (phospho-) proteome levels between nitrate and ammonium nutrition. Plant J 88: 717-734

\section{Mergner J, Frejno M, List M, Papacek M, Chen X, Chaudhary A, Samaras P, Richter S,} Shikata H, Messerer M, et al (2020) Mass-spectrometry-based draft of the Arabidopsis proteome. Nature 579: 409-414

Minato N, Komatsu K, Okano Y, Maejima K, Ozeki J, Senshu H, Takahashi S, Yamaji Y, Namba S (2014) Efficient foreign gene expression in planta using a plantago asiatica mosaic virus-based vector achieved by the strong RNA-silencing suppressor activity of TGBp1. Arch Virol 159: 885-896

Nikonorova N, Broeck LV den, Zhu S, Cotte B van de, Dubois M, Gevaert K, Inzé D, Smet ID (2018) Early mannitol-triggered changes in the Arabidopsis leaf (phospho)proteome reveal growth regulators. J Exp Bot 69: 4591-4607

Ott T (2017) Membrane nanodomains and microdomains in plant-microbe interactions. Curr Opin Plant Biol 40: 82-88

Perraki A, Binaghi M, Mecchia MA, Gronnier J, German-Retana S, Mongrand S, Bayer E, Zelada AM, Germain V (2014) StRemorin1.3 hampers Potato virus X TGBp1 ability to increase plasmodesmata permeability, but does not interfere with its silencing suppressor activity. Febs Lett 588: 1699-705

Perraki A, Cacas JL, Crowet JM, Lins L, Castroviejo M, German-Retana S, Mongrand S, Raffaele S (2012) Plasma Membrane Localization of Solanum tuberosum Remorin from Group 1, Homolog 3 Is Mediated by Conformational Changes in a Novel C-Terminal 
Anchor and Required for the Restriction of Potato Virus X Movement]. Plant Physiol 160: $624-37$

Perraki A, Gronnier J, Gouguet P, Boudsocq M, Deroubaix A-F, Simon V, GermanRetana S, Zipfel C, Bayer E, Mongrand S, et al (2018) REM1.3's phospho-status defines its plasma membrane nanodomain organization and activity in restricting PVX cell-to-cell movement. Plos Pathog 14: e1007378

Raffaele S, Bayer E, Lafarge D, Cluzet S, Retana SG, Boubekeur T, Leborgne-Castel N, Carde JP, Lherminier J, Noirot E, et al (2009) Remorin, a Solanaceae protein resident in membrane rafts and plasmodesmata, impairs potato virus X movement. Plant Cell 21: 154155

Raffaele S, Mongrand S, Gamas P, Niebel A, Ott T (2007) Genome-wide annotation of remorins, a plant-specific protein family: evolutionary and functional perspectives. Plant Physiol 145: 593-600

Schallus T, Fehér K, Sternberg U, Rybin V, Muhle-Goll C (2010) Analysis of the specific interactions between the lectin domain of malectin and diglucosides. Glycobiology 20: $1010-1020$

Schallus T, Jaeckh C, Feher K, Palma AS, Liu Y, Simpson JC, Mackeen M, Stier G, Gibson TJ, Feizi T, et al (2008) Malectin: a novel carbohydrate-binding protein of the endoplasmic reticulum and a candidate player in the early steps of protein N-glycosylation. Mol Biol Cell 19: 3404-14

Schindelin J, Arganda-Carreras I, Frise E, Kaynig V, Longair M, Pietzsch T, Preibisch S, Rueden C, Saalfeld S, Schmid B, et al (2012) Fiji: an open-source platform for biological-image analysis. Nat Methods 9: 676-82

Shevchenko A, Tomas H, Havli J, Olsen JV, Mann M (2006) In-gel digestion for mass spectrometric characterization of proteins and proteomes. Nat Protoc 1: 2856-2860

Shiu SH, Bleecker AB (2001) Plant receptor-like kinase gene family: diversity, function, and signaling. Sci Stke 2001: re22 
Son S, Oh CJ, Bae JH, Lee H, An CS (2015) GmREM1.1 and GmREM2.1, Which Encode the Remorin Proteins in Soybean, Have Distinct Roles during Root Nodule Development. Journal of Plant Biology 58: 17-25

Tinevez JY, Perry N, Schindelin J, Hoopes GM, Reynolds GD, Laplantine E, Bednarek SY, Shorte SL, Eliceiri KW (2016) TrackMate: An open and extensible platform for singleparticle tracking. Methods 115: 80-90

Tyanova S, Temu T, Sinitcyn P, Carlson A, Hein MY, Geiger T, Mann M, Cox J (2016)

The Perseus computational platform for comprehensive analysis of (prote)omics data. Nat Methods 13: 731-740

Wang L, Li H, Lv X, Chen T, Li R, Xue Y, Jiang J, Jin B, Baluska F, Samaj J, et al (2015) Spatiotemporal Dynamics of the BRI1 Receptor and its Regulation by Membrane Microdomains in Living Arabidopsis Cells. Mol Plant 8: 1334-49

Wei Z, Tan S, Liu T, Wu Y, Lei J-G, Chen Z, Friml J, Xue H-W, Liao K (2020) Plasmodesmata-like intercellular connections by plant remorin in animal cells. Biorxiv 791137

Xi L, Wu XN, Gilbert M, Schulze WX (2019) Classification and Interactions of LRR Receptors and Co-receptors Within the Arabidopsis Plasma Membrane - An Overview. Front Plant Sci 10: 472

Xiao Y, Stegmann M, Han Z, DeFalco TA, Parys K, Xu L, Belkhadir Y, Zipfel C, Chai J (2019) Mechanisms of RALF peptide perception by a heterotypic receptor complex. Nature 572: $270-274$

Yamada H, Hanaki N, Imamura A, Ueguchi C, Mizuno T (1998) An Arabidopsis protein that interacts with the cytokinin-inducible response regulator, ARR4, implicated in the HisAsp phosphorylay signal transduction. FEBS Lett 436: 76-80

Yoshida S, Parniske M (2005) Regulation of Plant Symbiosis Receptor Kinase through Serine and Threonine Phosphorylation*. J Biol Chem 280: 9203-9209 
Yue J, Li C, Liu Y, Yu J (2014) A remorin gene SiREM6, the target gene of SiARDP, from foxtail millet (Setaria italica) promotes high salt tolerance in transgenic Arabidopsis. PLOS ONE 9: e100772 
bioRxiv preprint doi: https://doi.org/10.1101/2021.01.28.428596; this version posted January 29, 2021. The copyright holder for this preprint (which was not certified by peer review) is the author/funder, who has granted bioRxiv a license to display the preprint in perpetuity. It is made available under aCC-BY-NC-ND 4.0 International license.

\section{Figures}

A

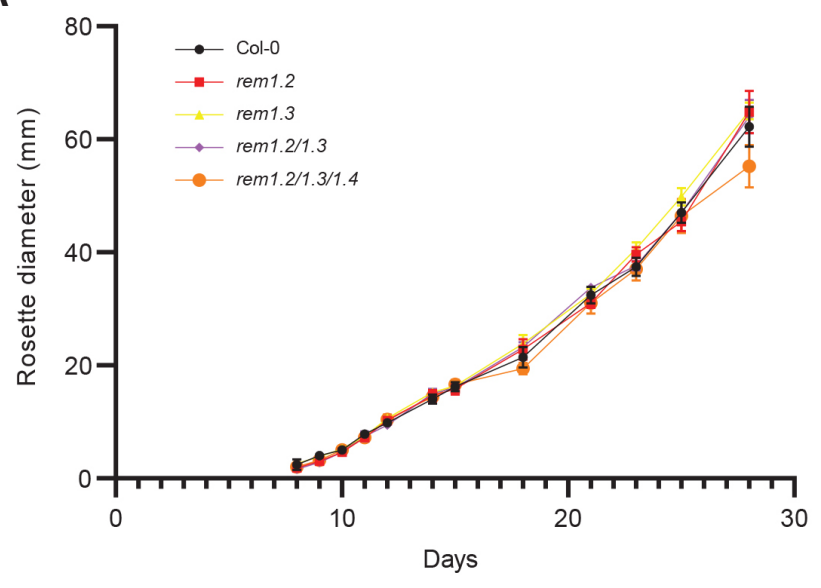

C

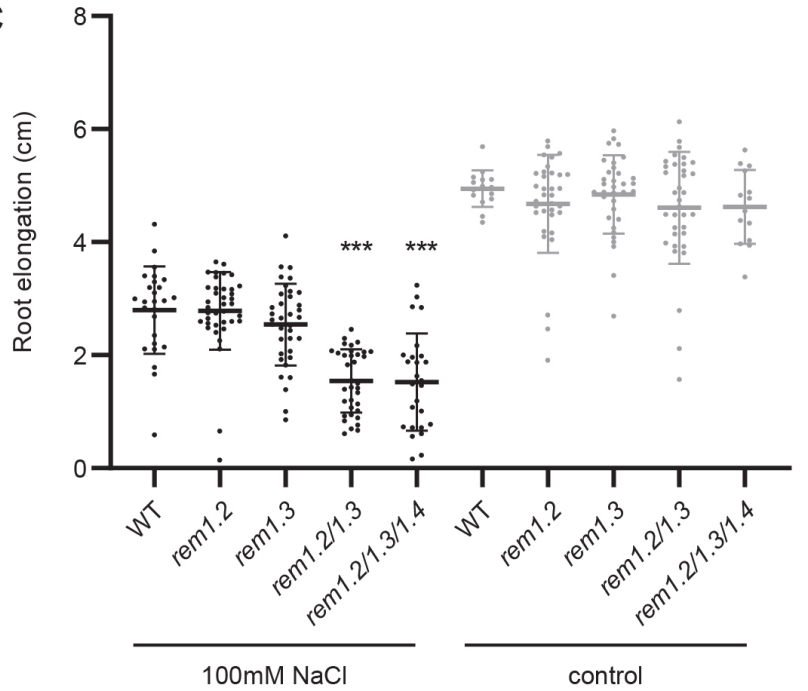

B
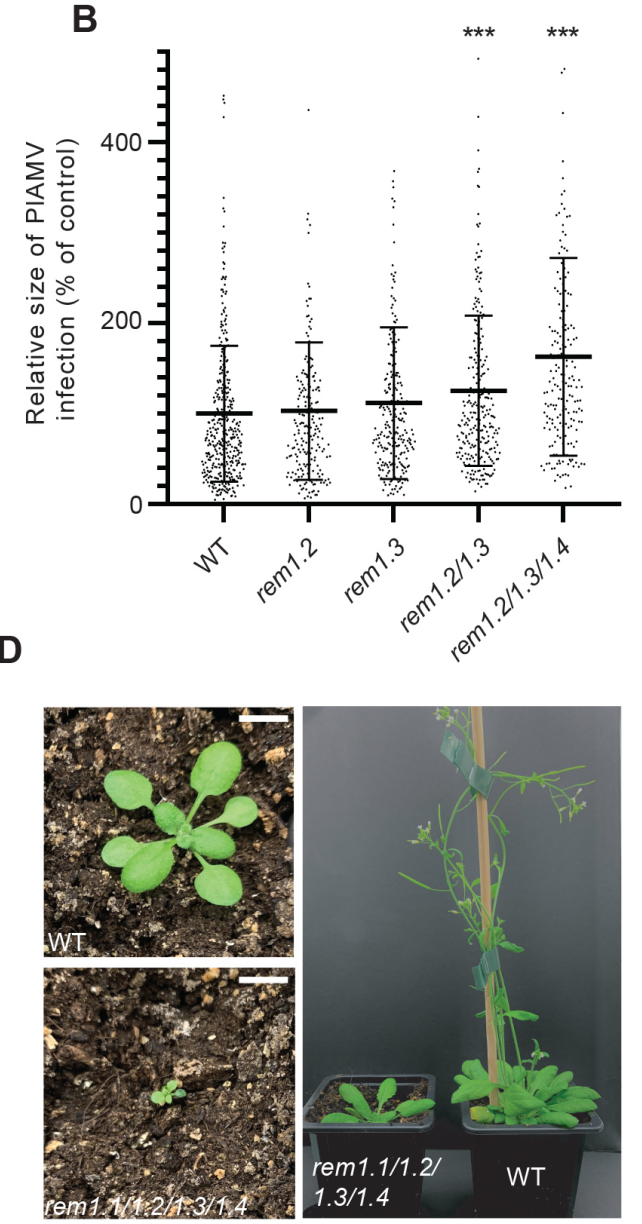

Fig. 1: Group 1 remorins are functionally redundant and essential for plant growth. (A) No growth phenotype was observed in rem1.2 (red), rem 1.3 (yellow) and rem1.2/1.3 (purple) mutants when scoring rosette diameter over time. (B) Assessing spreading of the Plantago asiatica mosaic virus (PlAMV) revealed an increased susceptibility of rem 1.2/1.3 double and rem1.2/1.3/1.4 triple mutants. (C) Plants were grown on $100 \mathrm{mM} \mathrm{NaCl}$ for seven days before root length was scored. While no differences were found in the controls (mock), growth of rem1.2/1.3 double and rem1.2/1.3/1.4 triple mutants was significantly reduced under salt stress. (D). A rem1. $1^{\mathrm{HET}} / 1.2^{\mathrm{HOM}} / 1.3^{\mathrm{HOM}} / 1.4^{\mathrm{HOM}}$ quadruple mutant generated by CRISPR-CAS9 showed a dwarf phenotype and the inability to set seeds. Scale bars represent $1 \mathrm{~cm}$. Significance was tested using a Dunnett's multiple comparisons test with $\mathrm{p}<0.001(* * *)$. 
A

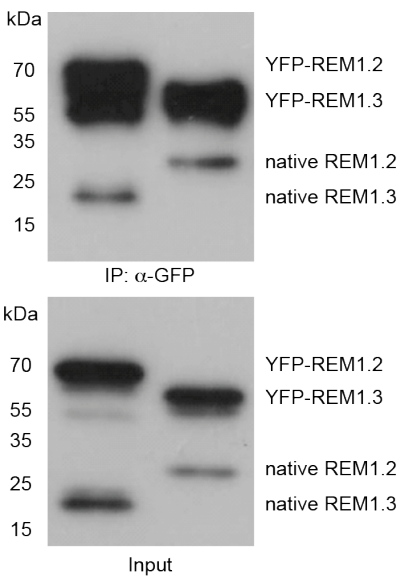

B

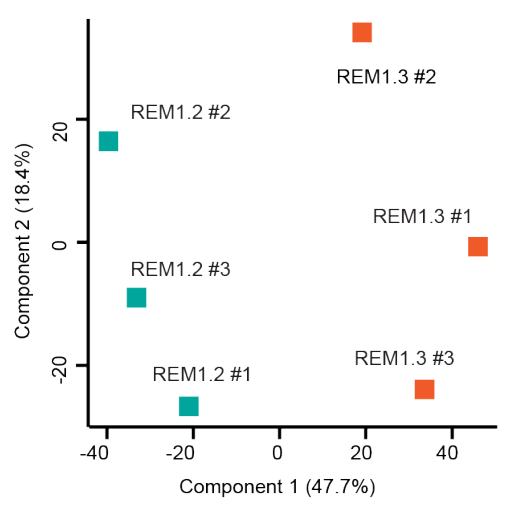

C

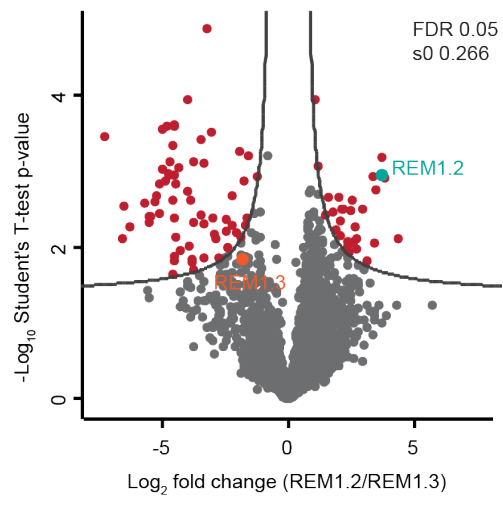

Fig. 2: REM1.2 and REM1.3 form a hetero-oligomeric complex in planta. (A) Co-immunoprecipitation of REM1.3 and REM1.2 in complemented rem1.2 and rem1.3 mutant lines, respectively, using GFP nanotraps and detecting endogenous REM1.2 and REM1.3 as well as the YFP fusion proteins by a specific antibody recognizing both proteins. (B) Principal component analysis (PCA) of REM1.2 and REM1.3 immuno-precipitation proteome analysis. (C) Volcano plot of REM1.2 and REM1.3 immuno-precipitation proteome fold changes and p-values. Significant different abundant proteins are depicted in red (Student's t-test, FDR < 0.05). REM1.2 and REM1.3 are depicted in blue and orange, respectively. 

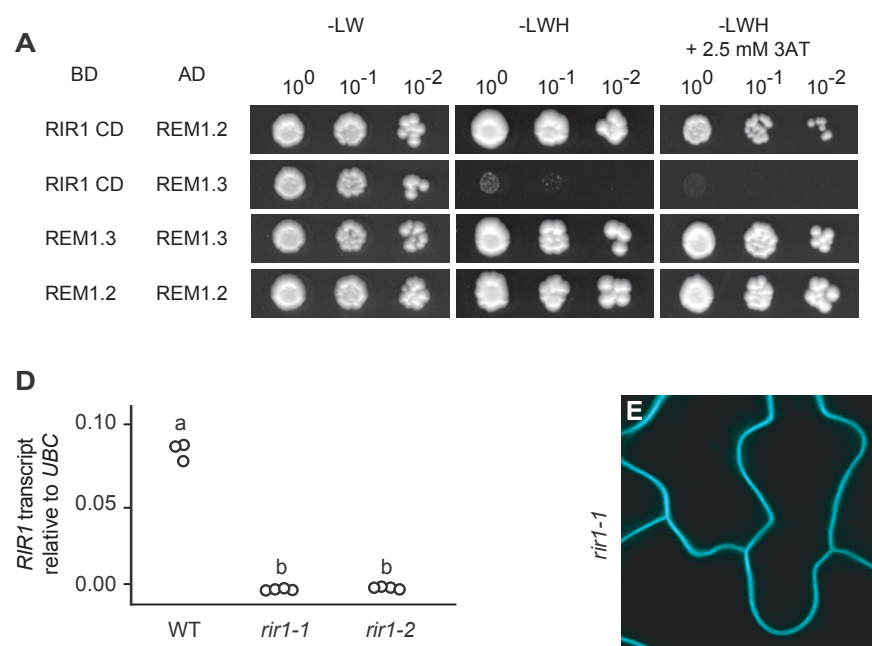

F

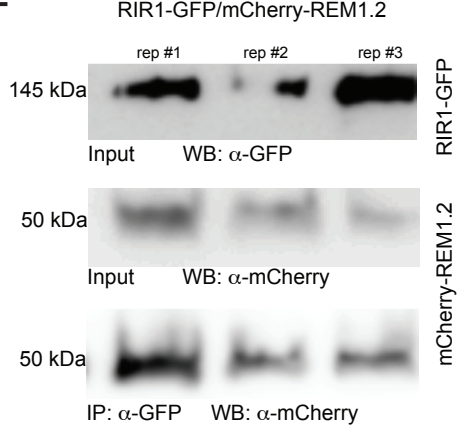

RIR1-GFP/mCherry-REM1.3
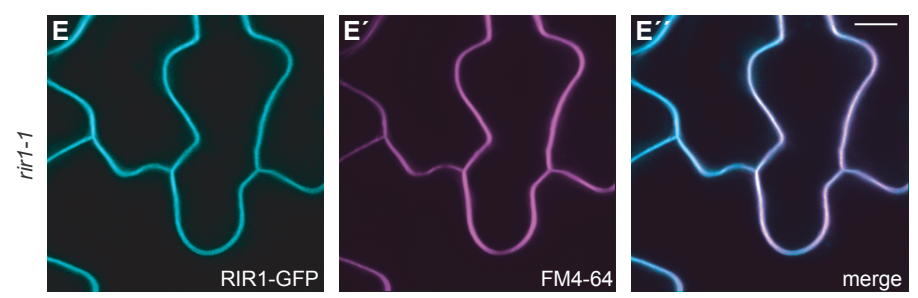
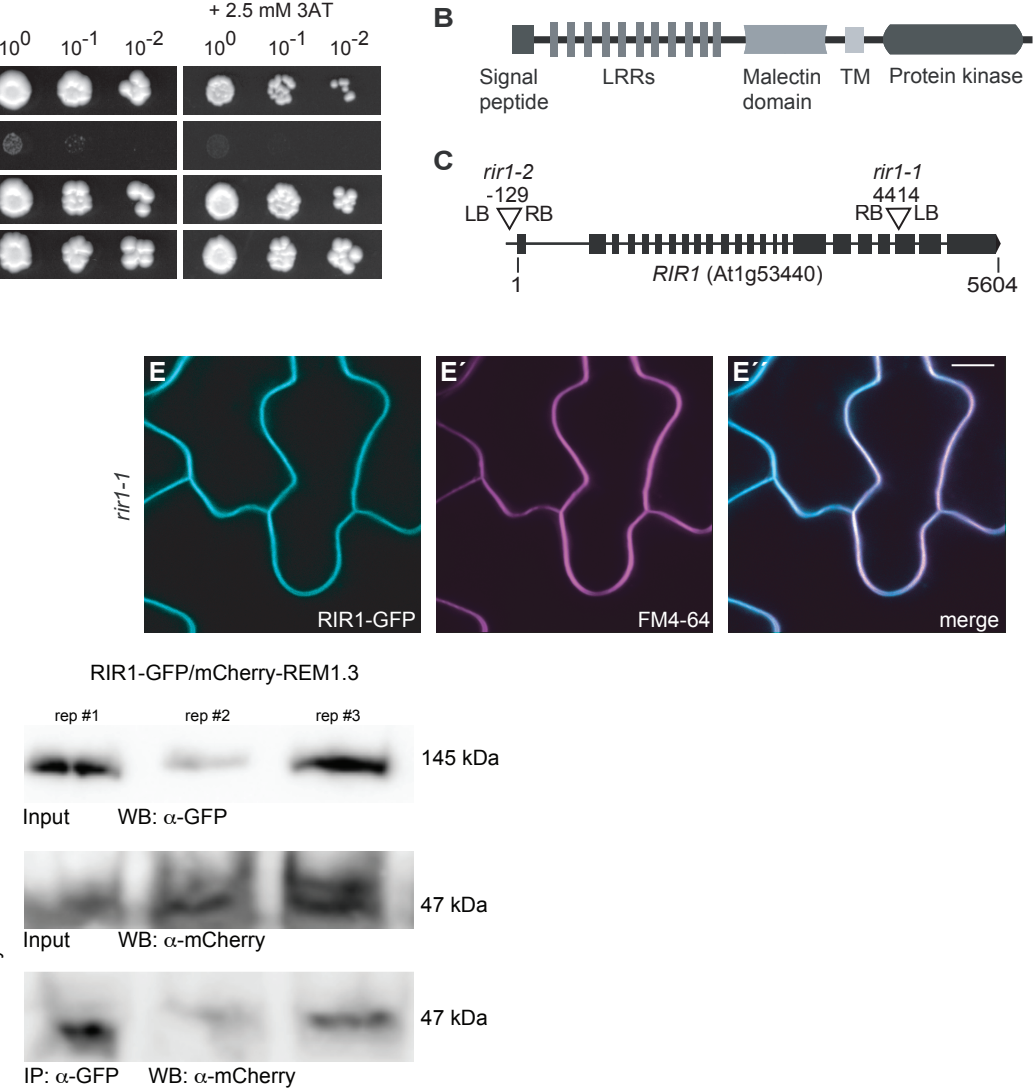

Fig. 3: Identification of the REMORIN-INTERACTING-RECEPTOR 1 (RIR1). (A) Screening for interaction partners for REM1.2 by independently co-expressing 55 isolated cytoplasmatic domains (CDs) of selected receptor-like kinases yielded in a strong interaction between RIR1 (At1g53440) with REM1.2 but not with REM1.3. Both remorin were able to homo-dimerize in yeast, demonstrating functionality of the clones. (B) Schematic illustration of the RIR1 protein structure with 11 leucin-rich repeats (LRRs) followed by a malectin domain, a transmembrane domain (TM) and a protein kinase domain. (C) Identification of two mutant alleles in public SALK mutant collection. Numbers indicate nucleotide positions. (D) Quantitative RealTime PCR confirmed the loss of transcript in both rirl mutant alleles. (E) Expressing of a fluorophore-tagged RIR1 driven by the endogenous RIRI promoter in the rirl-1 mutant background revealed plasma membrane localization of the receptor and co-localization with the membrane dye FM4-64 in five week old leaf epidermal cells (E'-E'). Scale bars indicate $10 \mu \mathrm{m}$. (F) Co-immunoprecipitation of RIR1 from independent rir1-1/ProRIR1-RIR1-GFP lines expression either a ProREM1.2-mCherry-REM1.2 or a ProREM1.3-mCherry-REM1.3 construct. background done in three independent replicates. REM1.2 and REM1.3 co-immunoprecipitating with RIR1 were detected with an $\alpha$-mCherry antibody. 

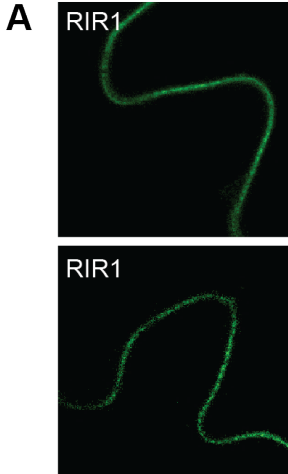

RIR1

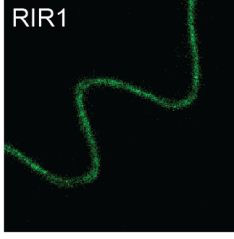

GFP channel
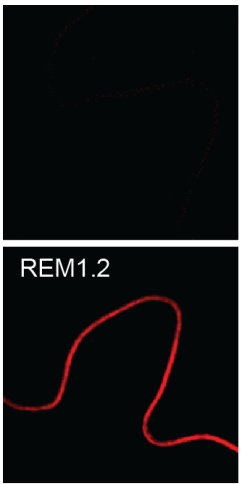

REM1.3

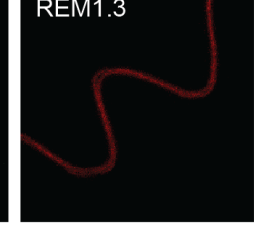

mCherry channel
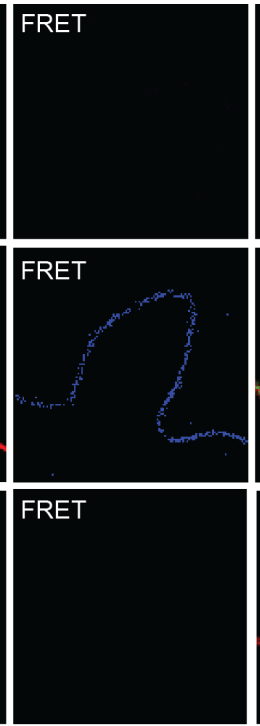

1
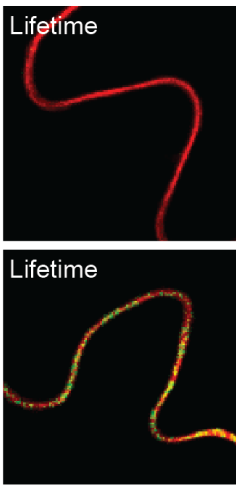

Lifetime

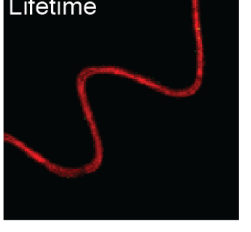

lifetime (ns)
B

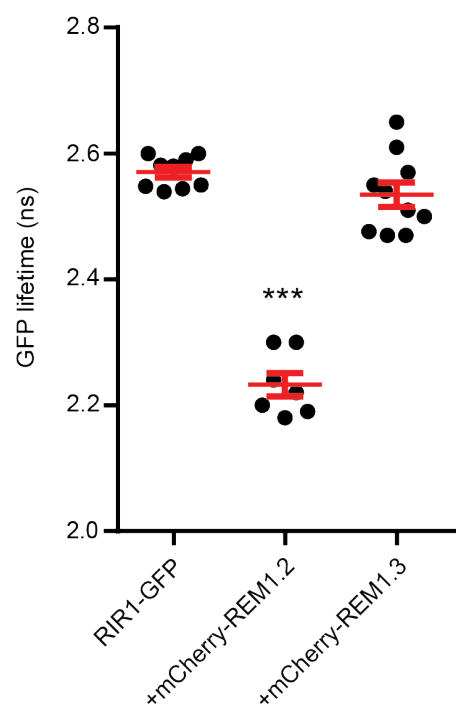

Fig. 4: Confirming the direct interaction between REM1.2 and RIR1 in stable transgenic Arabidopsis lines. Fluorescence Lifetime Imaging Microscopy-Förster Resonance Energy Transfer (FLIM-FRET) was applied to

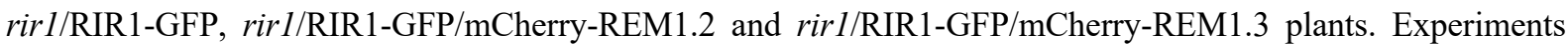
were performed on cotyledons of 5 days old seedlings. (A) Representative pictures on cells used for FRET analysis. (B) Quantification of the GFP lifetime. Significance was tested using a Dunn's multiple comparisons test with $\mathrm{p}<0.001(* * *)$. 
A

\begin{tabular}{|c|c|c|c|c|}
\hline & & $-\mathrm{LW}$ & $-\mathrm{LWH}$ & $-\mathrm{LWH}+2.5 \mathrm{mM} 3 \mathrm{AT}$ \\
\hline$A D$ & BD & $10^{-1}$ & $10^{-1} \quad 10^{-2}$ & $10^{0} \quad 10^{-1} \quad 10^{-2}$ \\
\hline RIR1 & REM1.2 & & 6 & E \\
\hline RIR1 $1^{D 881 N}$ & REM1.2 & D & 8 & (2) \\
\hline RIR1 & REM1.2 $2^{\mathrm{S} 11 / \mathrm{S} 13 \pi / 18 \mathrm{~T} 19 \mathrm{~A}}$ & 8 & क 9 & (c) \\
\hline RIR1 & REM1.2 $2^{\text {T41/T46A }}$ & e & & E 80 \\
\hline RIR1 & REM1.2 $2^{\text {T70A }}$ & e & 8 & $\theta \Leftrightarrow$ \\
\hline RIR1 & REM1.2 $2^{\mathrm{S} 11 \mathrm{~A}}$ & e & e) 4 & 0 \\
\hline RIR1 & REM1. $2^{\text {S13A }}$ & 88 & 0 क 0 & (c) \\
\hline RIR1 & REM1. $2^{\top 18 A}$ & $\bullet$ & 0 is $\cdots$ & 3 \\
\hline RIR1 & REM1.2 $2^{T 19 A}$ & 0 & $0 *$ & 0 \\
\hline REM1.2 & REM1.2 $2^{\mathrm{S} 11 / \mathrm{S} 13 / 118 / 19 \mathrm{~A}}$ & & 88 & 8 \\
\hline
\end{tabular}

B

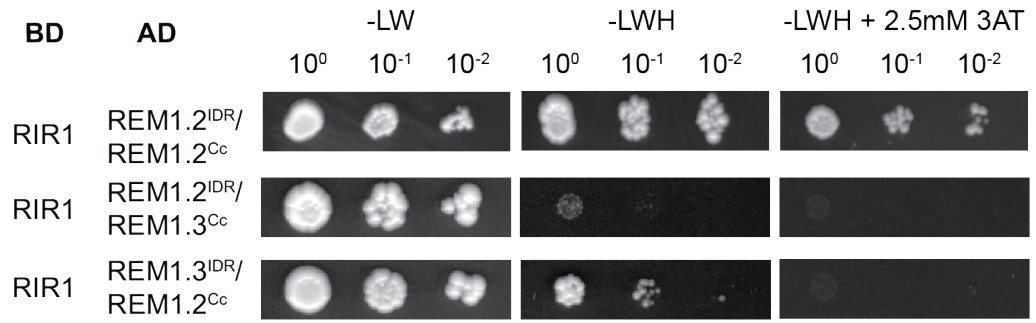

Fig. 5: Phosphorylation-dependent interaction between RIR1 and REM1.2 in yeast. (A) GAL4 Y2H assay of RIR1 wild type or kinase-dead (D811N) with REM1.2 wild type or phospho-ablative (S11/S13/T18/T19A, S11A, S13A, T18A, T19A, T41/T46A, T70A) mutants. (B) Testing chimeric remorin proteins where the intrinsically disordered N-terminal region (IDR) and the conserved coiled coil $(\mathrm{Cc})$ region were reciprocally exchanged between REM1.2 and REM1.3. Remorins were fused to the activation domain $(\mathrm{AD})$ and the kinases to the binding domain (BD) of GAL4. Transformants were grown on control medium (-LW) and selective medium (-LWH +/$2.5 \mathrm{mM} 3 \mathrm{AT}$ ) in three consecutive dilutions. $\mathrm{S}=$ serine, $\mathrm{T}=$ threonine, $\mathrm{L}=$ leucine, $\mathrm{W}=$ tryptophan, $\mathrm{H}=$ histidine, 3-AT = 3-amino-1,2,4-triazole. 
A

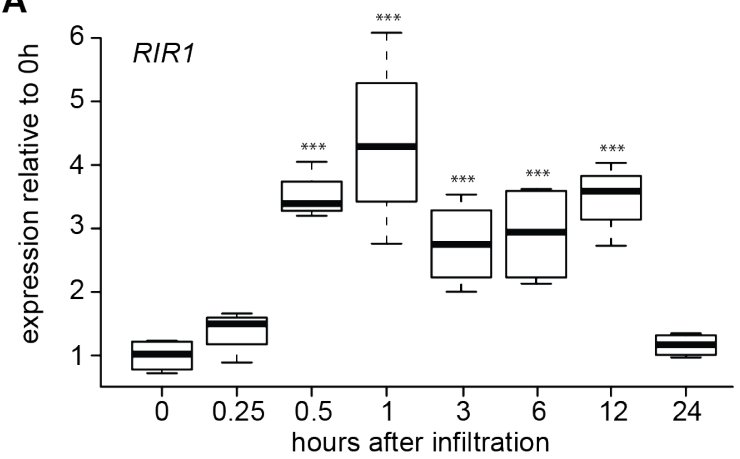

C

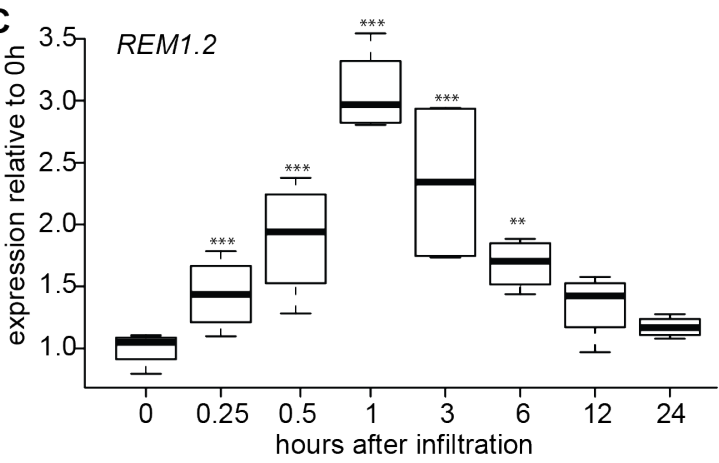

E

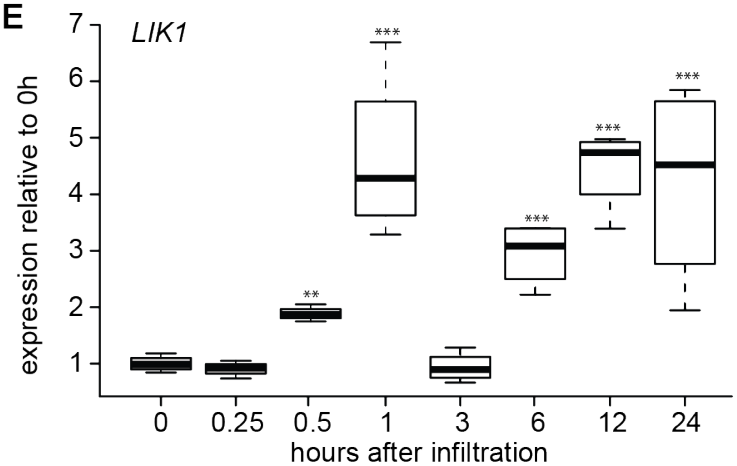

B
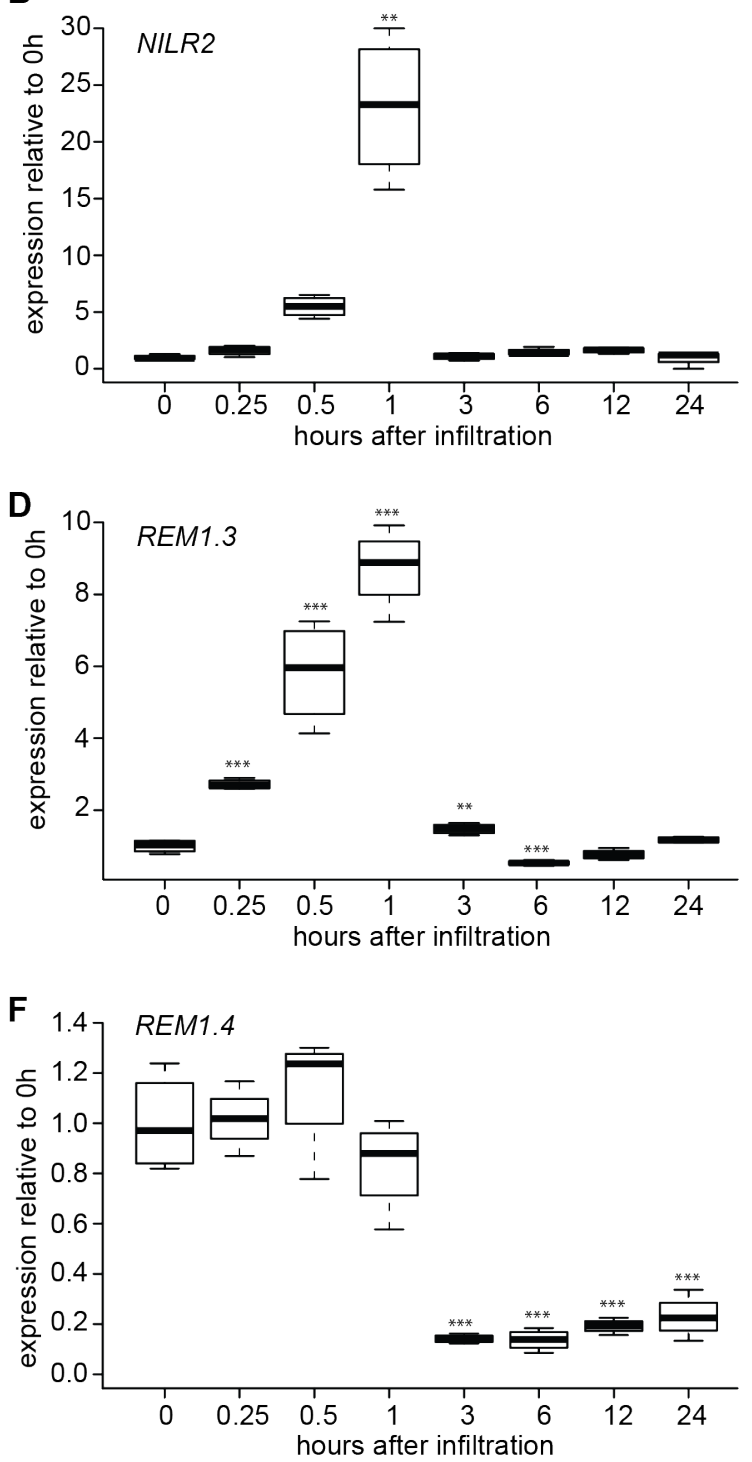

Fig. 6: RIR1, NILR2, REM1.2 and REM1.3 are up-regulated upon water infiltration. Five-week-old Col-0 leaves were infiltrated with $\mathrm{H}_{2} \mathrm{O}$ and harvested at the indicated time points $(\mathrm{n}=4)$. RIRl (A), NILR2 (B), REM1.2 (C), REM1.3 (D), LIKI (E) and REM1.4 (F) transcripts were normalized to UBC. Transcripts in the non-infiltrated control $(0 \mathrm{~h})$ were set to 1 , respectively. Statistical significance was assessed using one-way ANOVA followed by Dunnett's multiple comparison test $\left({ }^{* *} \mathrm{p} \leq 0.01, * * * \mathrm{p} \leq 0.001\right)$. 

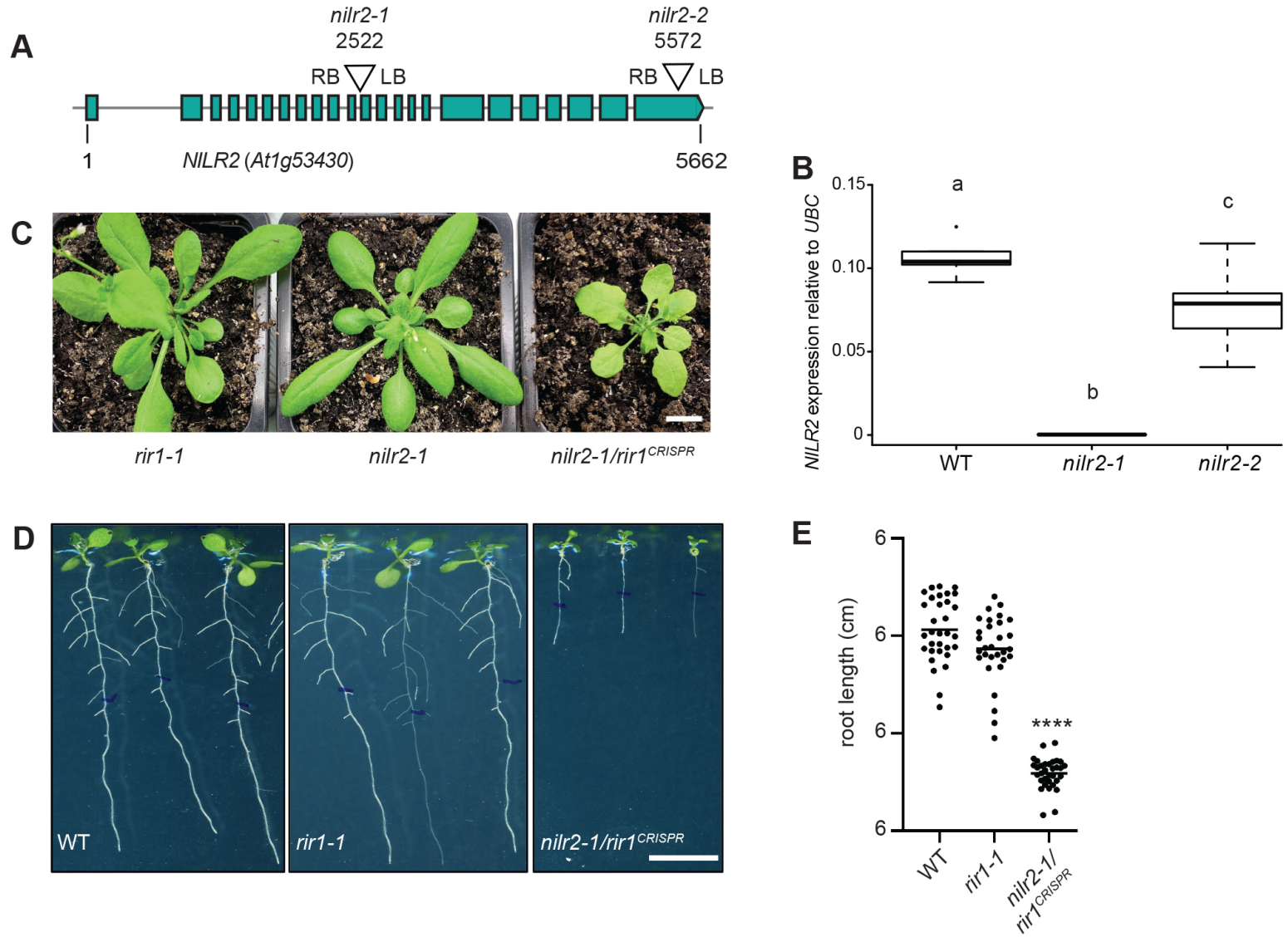

Fig. 7: RIR1 and NILR2 form a functionally redundant gene cluster. (A) Schematic illustration of the NILR2 gene (At1g53430) that is adjacent to the RIR1 locus. (B) Quantitative RealTime PCR on nilr2 alleles demonstrates nilr2-1 being a genetic knock-out while nilr2-2 transcripts were less strongly affected. Statistical significance for each transcript was assessed using ANOVA followed by Tukey HSD test $(\mathrm{p}<0.05)$. (C) While the rir $1-1$ allele did not show any growth phenotype, a rirl/nilr2 double mutant generated by introducing a CRISPR-CAS9-mediated $1725 \mathrm{bp}$ mutation in the RIR1 locus (nilr2-1/rirl ${ }^{C R I S P R}$ ) resulted in strongly retarded growth in seedlings. (D) In vitro growth phenotype scored on plants grown for ten days on MS agar plates. (E) Quantification of the nilr21/rir $1^{\text {CRISPR }}$ root phenotype. Statistical significance for each transcript was assessed using ANOVA followed by a Tukey multiple comparison test $(\mathrm{p}<0.0001)$. Scale bars indicate $1 \mathrm{~cm}$. 


\section{Supplemental Figures}
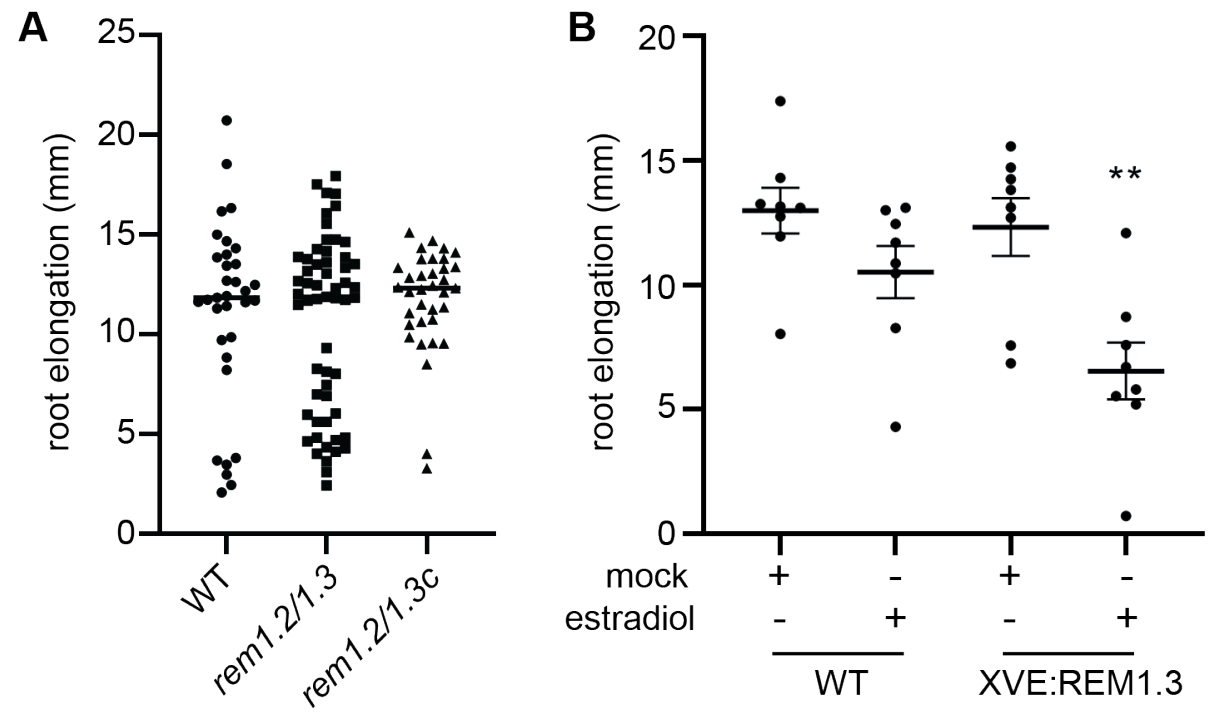

Figure S1: Assessing the growth phenotype of published remorin mutants under our growth conditions. (A) Root elongation of WT, rem1.2/ rem1.3 and rem1.2/rem1.3c (mutant published in Huang et al., 2019) over seven days. (B) Root elongation of WT and the published estradiol-inducible REM1.3 line XVE:REM1.3 three days after estradiol or mock treatment. Significance was tested using a Dunnett's multiple comparisons test with $\mathrm{p}<0.01(* *)$.

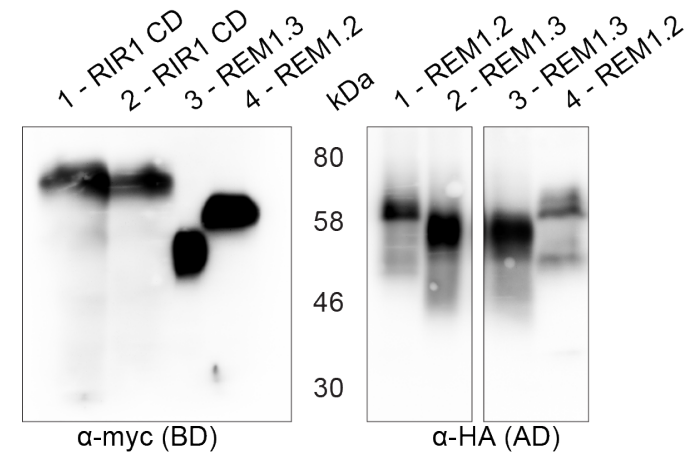

Fig. S2: (B) Expression analysis of yeast clones using immunoblot analysis. Activation domain (AD)-fused proteins were visualized with an $\alpha \mathrm{HA}$ antibody and binding domain (BD)-fused proteins with a $\alpha-$ myc antibody. $\mathrm{CD}=$ cytoplasmatic domain. 
A

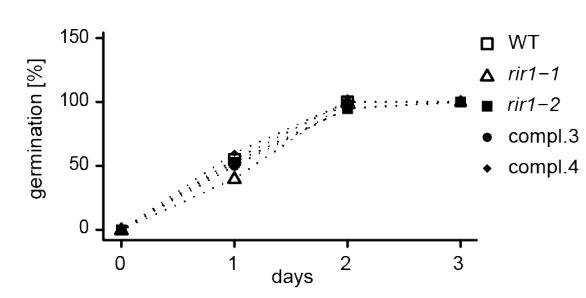

C

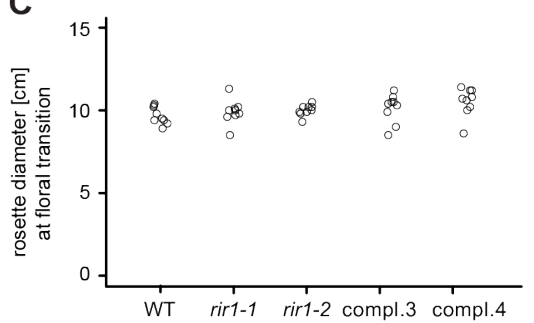

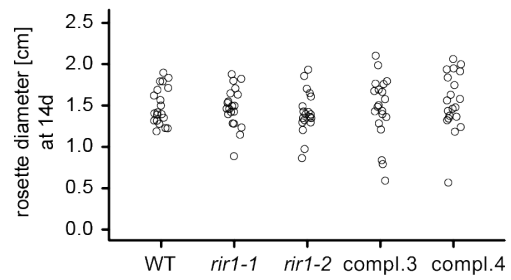

D

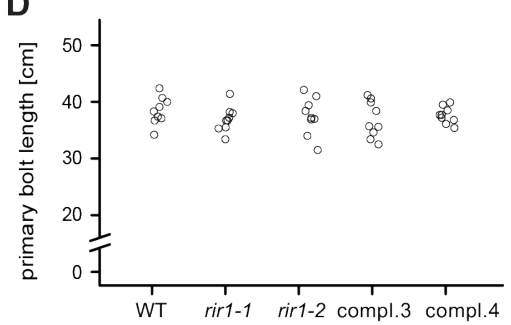

Fig. S3: rir1 mutants do not exhibit developmental defects. Different developmental stages were examined in Col-0 and rirl mutants grown under long-day conditions on plate $(1 / 2 \mathrm{MS}+1 \%$ sucrose $)(\mathrm{A}, \mathrm{B} ; \mathrm{n}=20)$ and on soil $(\mathrm{C}-\mathrm{F} ; \mathrm{n}=9)$. (A) Germination rates were scored on the indicated days. (B,C). The diameter of rosettes was measured in 14-day-old seedlings (B) and adult plants at the time of floral transition (C). The primary bolt length (D) was analyzed in seven-week-old plants. There was no significant difference between WT and the rirl mutants. Statistical significance was assessed using Fisher's exact test (A) or one-way ANOVA (B-D), respectively. 
A

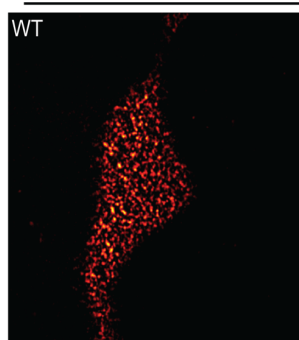

RIR1-GFP

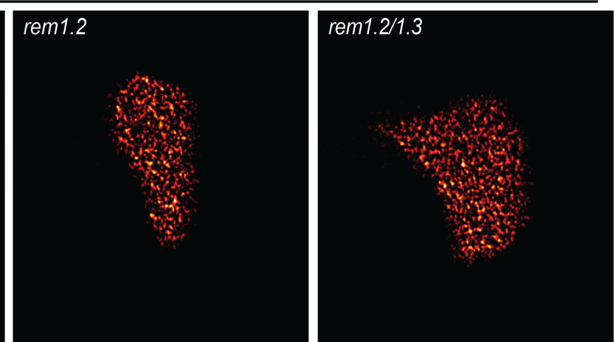

B

RIR1-GFP
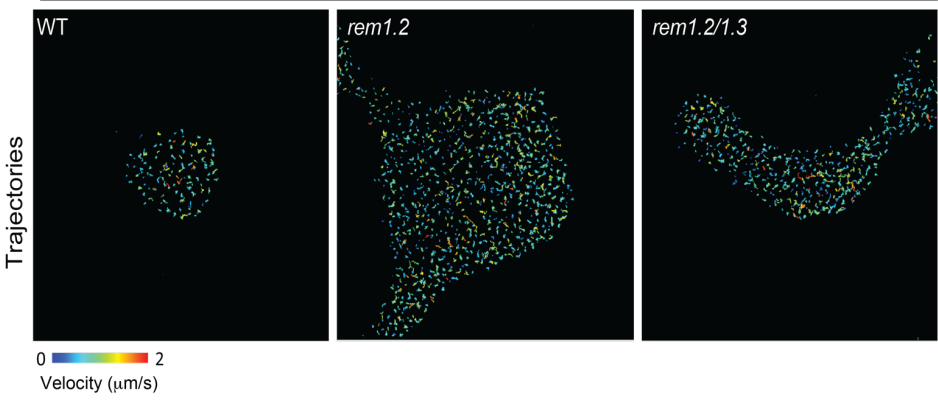

C
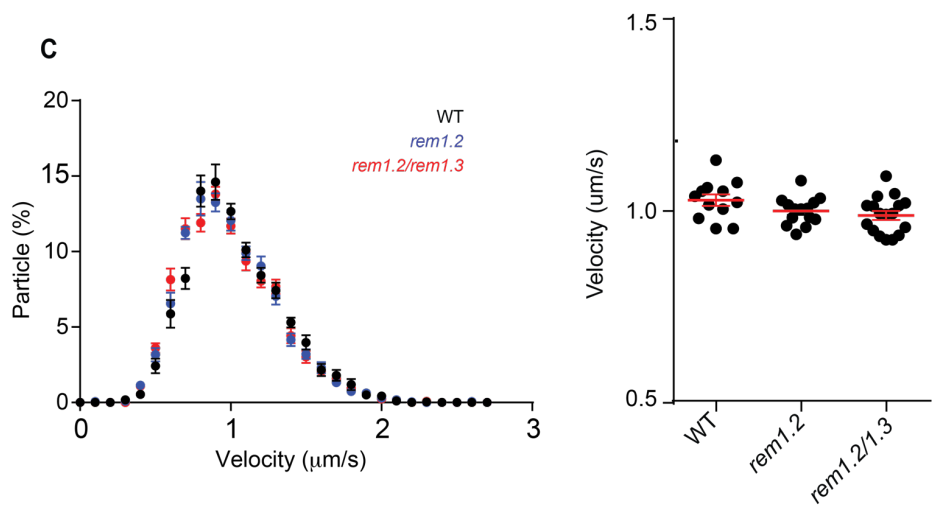

Fig. S4: RIR1-positive nanodomains form in a REM1.2/1.3-independent manner. (A) TIRFM on rirl/RIR1GFP, rem1.2/RIR1-GFP and rem1.2/rem1.3/RIR1-GFP. Experiments were performed on cotyledons of 5 days old seedlings. Image rendering: subtract background 20 rolling pixel and smoothing. (B) Single-particle tracking on rirl/RIR1-GFP, rem1.2/RIR1-GFP and rem1.2/rem1.3/RIR1-GFP. Experiment performed on cotyledons of 5 days old seedlings. (C) Quantification of RIR1-GFP single particle velocity. Mobility of RIR1-GFP is not altered in rem1.2 or rem1.2/rem1.3 mutants. 
A

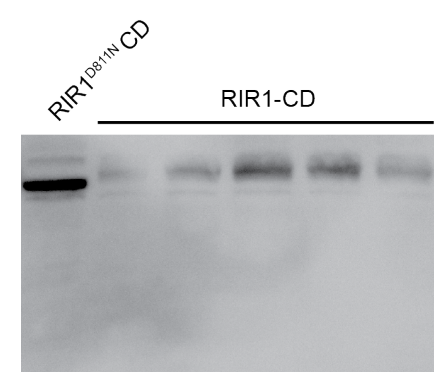

$\alpha$-myc (BD)

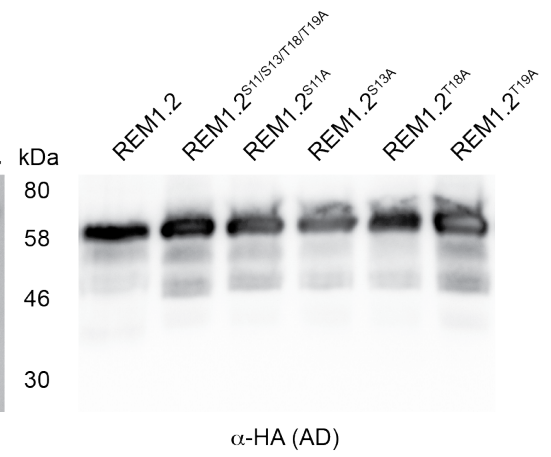

$\alpha-H A(A D)$
B

RIR1-CD kDa

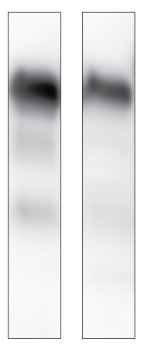

$\alpha$-myc (BD)

80

58

46
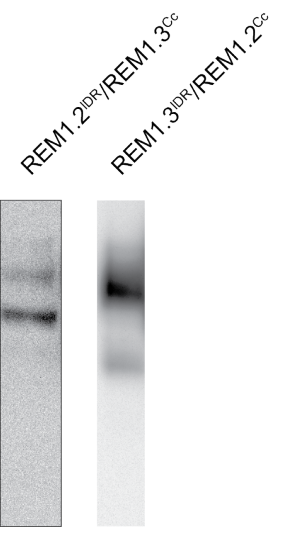

$\alpha-\mathrm{HA}(\mathrm{AD})$

Fig. S5: Western Blot analysis confirming the expression of clones that did not result in any interaction. (A) Blot supporting protein accumulations for Fig. 5A. (B) Blot supporting protein accumulations for Fig. 5B. Activation domain (AD)-fused proteins were visualized with an $\alpha$-HA antibody and binding domain (BD)-fused proteins with an $\alpha$-myc antibody. $\mathrm{CD}=$ cytoplasmic domain. Molecular weight is indicated in $\mathrm{kDa}$. 

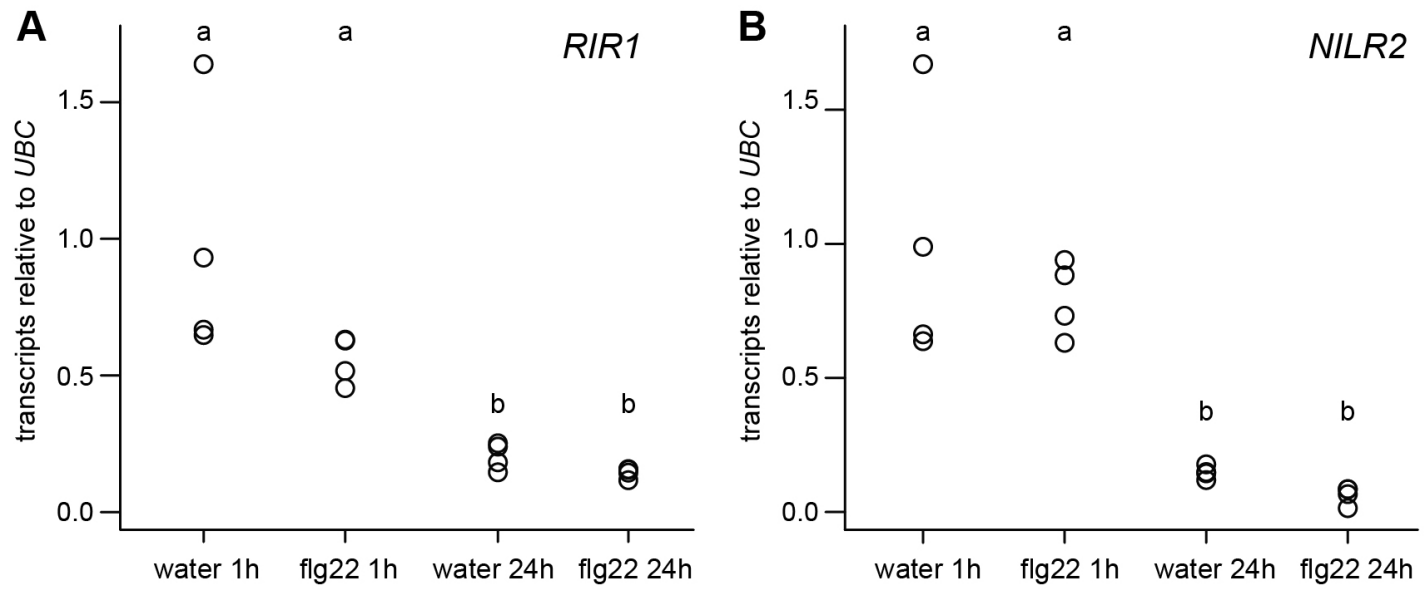

Fig. S6: RIR1 and NILR2 transcripts are differently regulated $1 \mathrm{~h}$ and $24 \mathrm{~h}$ after infiltration. Five-week-old Col-0 plants were infiltrated with $\mathrm{H}_{2} \mathrm{O}$ or flg22 and harvested $1 \mathrm{~h}$ and $24 \mathrm{~h}$ later. RIRl (A) and NILR2 (B) transcripts were analyzed using qPCR. Both transcripts were significantly lower $24 \mathrm{~h}$ after infiltration compared to $1 \mathrm{~h}$ after infiltration independent of the treatment. Statistical significance was assessed using one-way ANOVA followed by a Tukey HSD test $(\mathrm{p} \leq 0.005)$. 

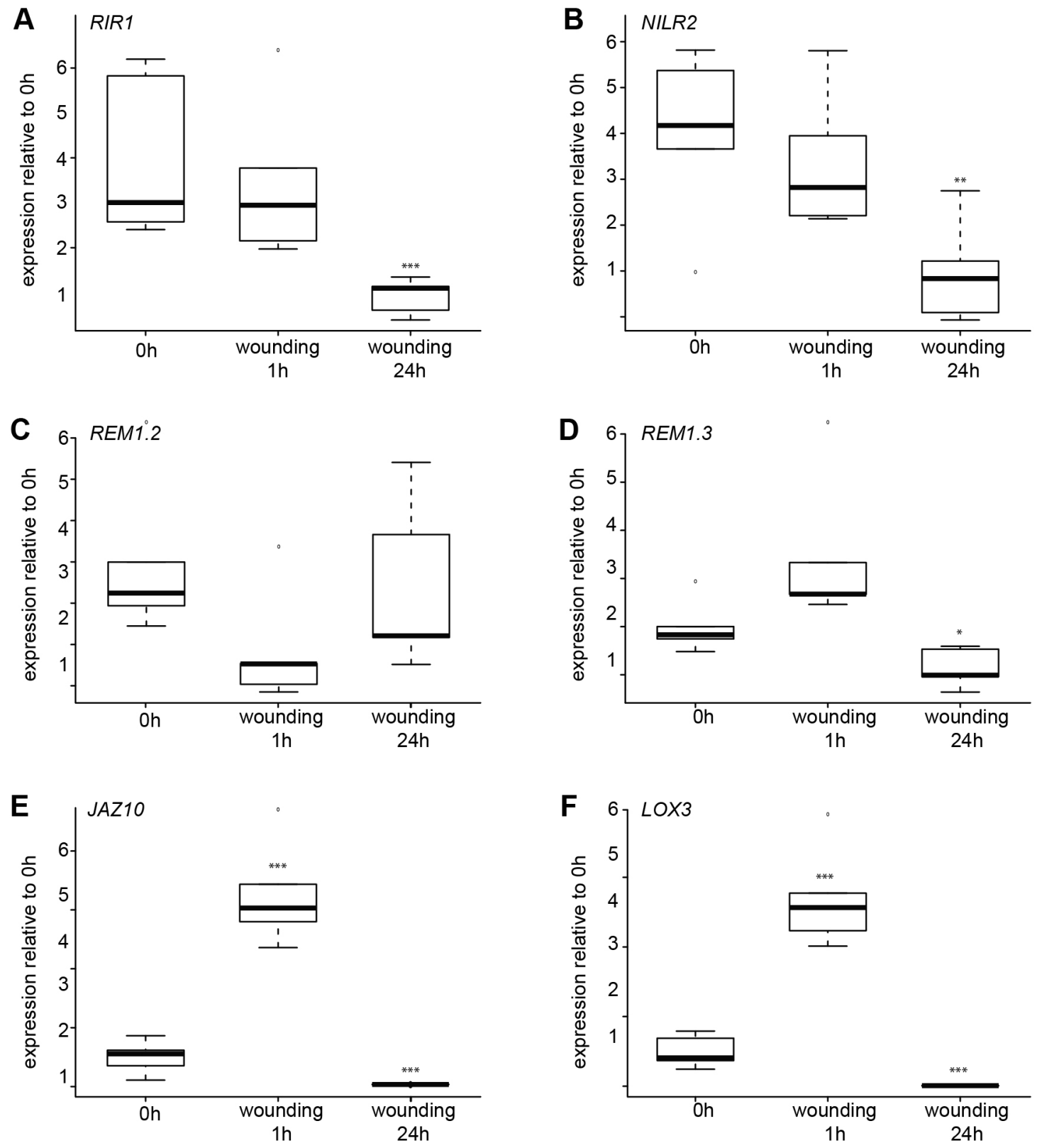

Fig. S7: RIR1, NILR2, REM1.2 and REM1.3 transcripts are not up-regulated upon wounding. Five-weekold WT Col-0 leaves were wounded with a needle and harvested at the indicated time points. RIRI (A), NILR2 (B), REM1.2 (C), REM1.3 (D), JAZ10 (E) and LOX3 (F) transcripts were normalized to UBC. The wounding marker genes $J A Z 10$ and $L O X 3$ were used as controls. Transcripts in the non-wounded control $(0 \mathrm{~h})$ were set to 1 , respectively. Statistical significance was assessed using one-way ANOVA followed by Dunnett's multiple comparison test $(* \mathrm{p} \leq 0.05, * * \mathrm{p} \leq 0.01, * * * \mathrm{p} \leq 0.001)$. 
A

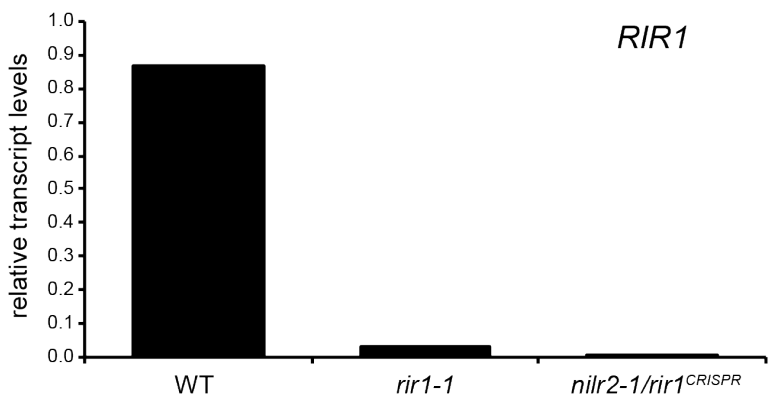

B

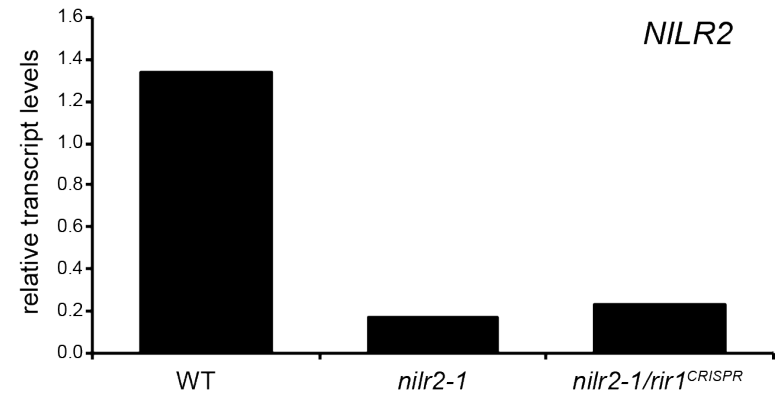

Fig. S8: CRISPR-CAS9-mediated mutation of the RIR1 locus in the nilr2-1 mutant background. (A) Transcripts of RIR1 in the rirl-1 and the nilr2-1/rirl ${ }^{\text {CRISPR }}$ mutant background. (B) Transcripts of NILR2 in the nilr2-1 and the nilr2-1/rir $1^{\text {CRISPR }}$ mutant background. Expression was determined by quantitative RealTime PCR and normalized to Ubiquitin. 